\title{
A RANDOM SPACE-TIME BIRTH PARTICLE METHOD FOR 2D VORTEX EQUATIONS WITH EXTERNAL FIELD
}

\author{
J. FONTBONA AND S. MÉLÉARD
}

\begin{abstract}
We consider incompressible 2d Navier-Stokes equations in the whole plane with external nonconservative forces fields. The initial data and external field are functions assumed to satisfy only slight integrability properties. We develop a probabilistic interpretation of these equations based on the associated vortex equation, in order to construct a numerical particle method to approximate the solutions. More precisely, we relate the vortex equation with additional term to a nonlinear process with random space-time birth, which provides a probabilistic description of the creation of vorticity. We then introduce interacting particle systems defined for a regularized interaction kernel, whose births are chosen randomly in time and space. By a coupling method, we show that these systems are approximations of the nonlinear process and obtain precise convergence estimates. From this result, we deduce a stochastic numerical particle method to obtain the vorticity and also to recover the velocity field. The results are either pathwise or of weak convergence, depending on the integrability of the data. We illustrate our results with simulations.
\end{abstract}

\section{INTRODUCTION}

The aim of this paper is to obtain an approximation particle method based on the creation of vorticity to simulate solutions of Navier-Stokes equations with nonconservative external forces. The Navier-Stokes equation for a homogeneous and incompressible fluid in the whole plane subject to an external force field $\mathbf{f}$ is given by

$$
\begin{aligned}
& \frac{\partial \mathbf{u}}{\partial t}+(\mathbf{u} \cdot \nabla) \mathbf{u}=\nu \Delta \mathbf{u}-\nabla \mathbf{p}+\mathbf{f} ; \\
& \operatorname{div} \mathbf{u}(t, x)=0 ; \quad \mathbf{u}(t, x) \rightarrow 0 \text { as }|x| \rightarrow \infty .
\end{aligned}
$$

Here, $\mathbf{u}$ denotes the velocity field, $\mathbf{p}$ is the pressure function and $\nu>0$ is the viscosity (constant) coefficient.

In absence of the external force field (or more generally, when $\mathbf{f}=\nabla \Psi$ is a conservative field), a probabilistic interpretation of (11) has been known since the work of Marchioro and Pulvirenti [16. The probabilistic approach to (11) is based on the associated vortex equation, i.e. the equation satisfied by the (scalar) vorticity field

Received by the editor November 6, 2006 and, in revised form, June 22, 2007.

2000 Mathematics Subject Classification. Primary 65C35, 76D17, 76M23; Secondary 82C22.

The first author was supported by Fondecyt Projects 1040689 and 1070743, Millennium Nucleus ICM P04-069-F, ECOS C05E02 and FONDAP Applied Mathematics.

The second author was supported by Fondecyt International Cooperation 7050142 and ECOS C05E02.

(C)2008 American Mathematical Society Reverts to public domain 28 years from publication 
$w:=$ curlu $\mathbf{u}$, which is interpreted as a generalized McKean-Vlasov equation associated with a nonlinear diffusion process. This process can also be obtained as the limit of interacting particle systems in mean field interaction, and this fact provides stochastic approximations of the vortex equation associated with (1). Convergence on the path space of these particles (or equivalently, propagation of chaos for the system) has been proved in more recent works of Méléard [17] and [18.

In this work, we extend that approach to the case of the Navier-Stokes equation with an external force field (11). The nonconservative external force introduces an additive term in the vortex equation. More precisely, the vorticity field $w=$ curl $\mathbf{u}$ satisfies the scalar equation

$$
\begin{aligned}
& \frac{\partial w}{\partial t}+(\mathbf{u} \cdot \nabla) w=\nu \Delta w+g ; \\
& w_{0}(x)=\text { curl } \mathbf{u}(0, x) .
\end{aligned}
$$

The external field $g=$ curlf can be physically interpreted as the creation of vorticity. In order to provide a probabilistic description of this phenomenon, we relate this equation to a nonlinear process with random birth in space and time, according to a law related to the initial vorticity $w_{0}$ and the external field $g$.

We will first consider a mollified setting, working with regularized versions of the Biot-Savart kernel and adapting classic McKean-Vlasov techniques to prove the pathwise existence and uniqueness of a mollified nonlinear process. The family of its time-marginal laws weighted by some function of the "space-time initial data" gives a solution of the mollified vortex equation. We then construct a stochastic interacting particle system whose births are chosen randomly in time and space and prove propagation of chaos and its convergence to the mollified nonlinear process.

Then, we remove the regularization parameter, first under the assumption that the data $w_{0}$ and $g$ are $L^{1} \cap L^{p}$ functions (for $p>\frac{4}{3}$ ), in order to deal with the singularity of the kernel $K$ (this choice is suggested by the continuity properties of the Biot and Savart operator). We take advantage of the volume preserving property of the stochastic flow associated with the mollified nonlinear process to obtain uniform $L^{p}$ estimates. We deduce the existence of a global mild solution of the vortex equation, and by analytic techniques we prove uniqueness and regularity of this solution. Such regularity is essential to get pathwise existence and uniqueness of the nonlinear process.

Moreover, we obtain pathwise convergence for the particle system in a strong norm, improving previous results on the stochastic vortex method. Some convergence estimates are given. We deduce an approximation result for the velocity field $\mathbf{u}$ at an explicit rate, which also enlightens the case where $\mathbf{f}=0$.

We then extend these results to the $L^{1}$-only initial condition and external field, which are the natural probabilistic assumptions. The analytical part generalizes to the case $g \neq 0$ some arguments of Ben-Artzi [1 and Brezis [5] when $g=0$. We obtain as before existence, uniqueness and regularity of the mild solution of the vortex equation, existence and uniqueness in law for the nonlinear process, together with convergence in law of the particle approximations. The explosion of the solution at time 0 prevents us in this case from obtaining pathwise results and stronger convergence.

In the last section, we develop a numerical example, simulating vortices created randomly in space and time. The law of these random births is computed from the 
initial condition and the additive term curlf , as described in the theoretical setup. Some numerical experiments are presented.

Let us point out that deterministic vortex creation methods have been developed for bounded domains [9], but such creations are related to the boundary condition (see also 14 for a probabilistic point of view). From a numerical point of view, an interesting feature of our method is that it does not necessitate the use of a grid. It is also worth noting that to our knowledge, no numerical method, either stochastic or deterministic, has so far been developed in the context of the whole plane and for general external force fields. We expect that the particle method we propose will be of interest for numerical applications.

Throughout the paper we shall need the following notation and the version of Gronwall's lemma quoted below (and proved e.g. in [10]):

- $C^{1,2}$ is the set of real valued functions on $[0, T] \times \mathbb{R}^{2}$ with continuous derivatives up to the first order in $t \in[0, T]$ and up to the second order in $x \in \mathbb{R}$. $C_{b}^{1,2}$ is the subspace of bounded functions in $C^{1,2}$ with bounded derivatives.

- $\mathcal{D}$ is the space of of infinitely differentiable functions on $\mathbb{R}^{2}$ having compact support.

- For all $1 \leq p \leq \infty$ we denote by $L^{p}$ the space $L^{p}\left(\mathbb{R}^{2}\right)$ of real valued functions on $\mathbb{R}^{2}$. By $\|\cdot\|_{p}$ we denote the corresponding norm and $p^{*}$ stands for the Hölder conjugate of $p$. We write $W^{i, p}=W^{i, p}\left(\mathbb{R}^{2}\right)$ for the Sobolev space of functions in $L^{p}$ with partial derivatives up to the $i$-th order in $L^{p}$.

- $C$ and $C(T)$ are finite positive constants that may change from line to line.

Lemma 1.1. Let $k:[0, T] \rightarrow \mathbb{R}_{+}$be a bounded measurable function and $C, A \geq 0$ and $\theta>0$ constants such that

$$
k(t) \leq A+C \int_{0}^{t}(t-s)^{\theta-1} k(s) d s
$$

for all $t \leq T$. Then, $\sup _{t \leq T} k(t) \leq C(T) A$ for some $C(T)>0$ not depending on $A$.

\section{The VORTEX EQUATION WITH EXTERNAL FORCE AND ITS PROBABILISTIC INTERPRETATION}

Is is well known that given $\mathbf{u}$ a regular solution of the Navier-Stokes equation with external force (1), the vorticity function $w=$ curl $\mathbf{u}$ satisfies equation (2), where $g=$ curl $\mathbf{f}$. By the divergence free property of $\mathbf{u}$ and the so-called Biot and Savart law, we can write $\mathbf{u}=K * w$ where

$$
K(x)=\frac{1}{2 \pi} \frac{\left(-x_{2}, x_{1}\right)}{|x|^{2}}, \quad x \in \mathbb{R}^{2} \backslash\{0\},
$$

is the so-called Biot-Savart kernel in $\mathbb{R}^{2}$. For these facts and further background on vorticity, we refer for instance to Chorin and Marsden [8], Ch. 1, or Bertozzi and Majda [2.

We will fix for all the sequels an arbitrary finite time interval $[0, T]$.

In view of our probabilistic interpretation of equation (2), it will be natural to assume that the functions $w_{0}: \mathbb{R}^{2} \rightarrow \mathbb{R}$ and $g: \mathbb{R}_{+} \times \mathbb{R}^{2} \rightarrow \mathbb{R}$ satisfy the minimal integrability hypothesis:

- $w_{0} \in L^{1}\left(\mathbb{R}^{2}\right)$.

- $g \in L^{1}\left([0, T] \times \mathbb{R}^{2}\right)$. 
We denote by $\|g\|_{1, T}$ the $L^{1}$-norm of $g$ on $[0, T] \times \mathbb{R}^{2}$ :

$$
\|g\|_{1, T}=\int_{0}^{T} \int_{\mathbb{R}^{2}}|g(s, x)| d x d s .
$$

We are interested in weak solutions of (2) defined as follows.

Definition 2.1. A measurable function $w:[0, T] \times \mathbb{R}^{2} \rightarrow \mathbb{R}$ is a weak solution of the vortex equation (2) with initial condition $w_{0}$ and external field $g$ on the interval $[0, T]$, if

$$
\int_{[0, T] \times \mathbb{R}^{2}}\left|K * w_{t}(x)\right|\left|w_{t}(x)\right| d x d t<\infty,
$$

and for every function $\phi \in C_{b}^{1,2}\left([0, T] \times \mathbb{R}^{2}\right)$ and $t \leq T$,

$$
\begin{gathered}
\int_{\mathbb{R}^{2}} \phi(t, x) w_{t}(x) d x=\int_{\mathbb{R}^{2}} \phi(0, x) w_{0}(x) d x+\int_{0}^{t} \int_{\mathbb{R}^{2}} \phi(s, x) g_{s}(x) d x d s \\
\quad+\int_{0}^{t} \int_{\mathbb{R}^{2}}\left[\frac{\partial \phi(s, x)}{\partial s}+\nu \triangle \phi(s, x)+\left(K * w_{s}\right)(x) \nabla \phi(s, x)\right] w_{s}(x) d x .
\end{gathered}
$$

For some analytic purposes we shall also need to deal with a mild form of equation (21). We denote by

$$
G_{t}^{\nu}(x):=(4 \pi \nu t)^{-1} e^{-|x|^{2} / 4 \nu t}
$$

the heat kernel in $\mathbb{R}^{2}$. The following are well known estimates, that are obtained using Young's inequality and the Gaussian bound for the space derivatives of the heat kernel (see e.g. [11]).

Lemma 2.2. Let $m \in[1, \infty]$ and $l \geq m$. There exist constants $c(m, l), c^{\prime}(m, l)>0$ such that for all $f \in L^{m}$

$$
\left\|G_{t}^{\nu} * f\right\|_{l} \leq c(m, l) t^{\frac{1}{l}-\frac{1}{m}}\|f\|_{m} \text { and }\left\|\nabla G_{t}^{\nu} * f\right\|_{l} \leq c^{\prime}(m, l) t^{-\frac{1}{2}+\frac{1}{l}-\frac{1}{m}}\|f\|_{m} .
$$

Definition 2.3. A measurable function $w:[0, T] \times \mathbb{R}^{2} \rightarrow \mathbb{R}$ is called a mild solution of the vortex equation with external field $g$ if condition (3) holds and

$$
w_{t}(x)=G_{t}^{\nu} * w_{0}(x)+\int_{0}^{t} G_{t-s}^{\nu} * g_{s}(x) d s+\int_{0}^{t} \nabla G_{t-s}^{\nu} *\left[\left(K * w_{s}\right) w_{s}\right](x) d s
$$

for all $t \in[0, T]$.

Remark 2.4. By taking for each fixed $t>0$ and $\psi \in \mathcal{D}$ in equation (4) the function $\phi_{t}(s, x):=G_{t-s}^{\nu} * \psi(x)$ (which solves on $\left[0, t\left[\times \mathbb{R}^{2}\right.\right.$ the heat equation with final condition $\psi$ ), and using Fubini's theorem (thanks to (3)), we see that weak solutions are always mild ones.

Now we shall relate the term involving the external field $g$ with the creation of vorticity in the whole space, and interpret equation (2) as a generalized McKeanVlasov equation, by introducing a nonlinear process with random space-time birth.

In Jourdain and Méléard [14, creation of vorticity on the boundary of a bounded domain is shown to be related to a vortex equation with Neumann's condition on the boundary, and an analogous probabilistic setup is developed in the bounded domain. Here, working in the whole space prevents us from using similar techniques. 
Let us define the probability measure $P_{0}(d t, d x)$ on $[0, T] \times \mathbb{R}^{2}$ by

$$
\begin{aligned}
P_{0}(d t, d x) & =\delta_{0}(d t) \frac{\left|w_{0}(x)\right|}{\left\|w_{0}\right\|_{1}+\|g\|_{1, T}} d x+\frac{\left|g_{t}(x)\right|}{\left\|w_{0}\right\|_{1}+\|g\|_{1, T}} d x d t \\
& =\delta_{0}(d t) \bar{\omega}_{0}(x) d x+\bar{g}_{t}(x) d x d t
\end{aligned}
$$

together with the scalar weight function

$$
h(t, x)=\mathbf{1}_{\{t=0\}} \frac{w_{0}(x)}{\left|w_{0}(x)\right|}\left(\left\|w_{0}\right\|_{1}+\|g\|_{1, T}\right)+\frac{g_{t}(x)}{\left|g_{t}(x)\right|}\left(\left\|w_{0}\right\|_{1}+\|g\|_{1, T}\right) \mathbf{1}_{\{t>0\}}
$$

with the convention " $\frac{0}{0}=0 "$ and $\mathbf{1}$ denoting the indicator function. We note that $|h(t, x)|=\left\|w_{0}\right\|_{1}+\|g\|_{1, T}$ or 0 .

Remark 2.5. For any measurable bounded function $\phi$ on $[0, T] \times \mathbb{R}^{2}$,

$$
\int_{[0, T] \times \mathbb{R}^{2}} \phi(t, x) h(t, x) P_{0}(d x, d t)=\int_{\mathbb{R}^{2}} \phi(0, x) w_{0}(x) d x+\int_{[0, T] \times \mathbb{R}^{2}} \phi(t, x) g_{t}(x) d x d t .
$$

In the sequel, $\left(\tau,\left(X_{t}\right)_{t \in[0, T]}\right)$ denotes the canonical process on the space $\mathcal{C}_{T}:=$ $[0, T] \times C\left([0, T], \mathbb{R}^{2}\right)$. With each probability measure $Q$ on $\mathcal{C}_{T}$ we associate a flow of signed measures $\left(\tilde{Q}_{t}\right)_{t \in[0, T]}$ on $\mathbb{R}^{2}$, defined for bounded measurable functions $f: \mathbb{R}^{2} \rightarrow \mathbb{R}$ by

$$
\tilde{Q}_{t}(f)=E^{Q}\left(f\left(X_{t}\right) h\left(\tau, X_{0}\right) \mathbf{1}_{t \geq \tau}\right) .
$$

The total mass of $\tilde{Q}_{t}$ is bounded by $\left\|w_{0}\right\|_{1}+\|g\|_{1, T}$. Moreover, if $Q \circ\left(X_{t}\right)^{-1}$ has a density, say $\rho_{t}$, then so does $\tilde{Q}_{t}$.

We then denote the density of $\tilde{Q}_{t}$ by $\tilde{\rho}_{t}(x)$, and, when they exist, take versions of $\rho_{t}(x)$ and $\tilde{\rho}_{t}(x)$ that are measurable in $(t, x)$.

Definition 2.6. A probability measure $P$ on $\mathcal{C}_{T}$ is a solution to the nonlinear martingale problem (MP) if

- $\quad P \circ\left(\tau, X_{0}\right)^{-1}=P_{0}$ and $\tilde{P}_{t}$ has bi-measurable densities $\tilde{\rho}_{t}(x)$ for $(t, x) \in[0, T] \times \mathbb{R}^{2}$

- $f\left(t, X_{t}\right)-f\left(\tau, X_{0}\right)$ $-\int_{0}^{t}\left[\frac{\partial f}{\partial s}\left(s, X_{s}\right)+\nu \triangle f\left(s, X_{s}\right)+K * \tilde{\rho}_{s}\left(X_{s}\right) \nabla f\left(s, X_{s}\right)\right] \mathbf{1}_{s \geq \tau} d s$,

$0 \leq t \leq T$, is a continuous $P$-martingale for all $f \in \mathcal{C}_{b}^{1,2}$ w.r.t. the filtration $\mathcal{F}_{t}=\sigma\left(\tau,\left(X_{s}\right), s \leq t\right)$.

The link between this problem and equation (2) is the following.

Lemma 2.7. Assume that the problem (MP) has a solution $P$ which satisfies

$$
\int_{[0, T] \times \mathbb{R}^{2}}\left|K * \tilde{\rho}_{t}(x)\right|\left|\tilde{\rho}_{t}(x)\right| d x d t<\infty .
$$

Then $w:=\tilde{\rho}$ is a weak solution of the vortex equation (2) with external field $g$. 
Proof. Since the variable $h\left(\tau, X_{0}\right) \mathbf{1}_{\{\tau \leq t\}}$ is measurable with respect to $\mathcal{F}_{0}$, by definition of (MP) the processes

$$
\begin{aligned}
& f\left(t, X_{t}\right) h\left(\tau, X_{0}\right) \mathbf{1}_{\{\tau \leq t\}}-f\left(\tau, X_{0}\right) h\left(\tau, X_{0}\right) \mathbf{1}_{\{\tau \leq t\}} \\
& \quad-\int_{0}^{t}\left[\frac{\partial f}{\partial s}\left(s, X_{s}\right)+\nu \triangle f\left(s, X_{s}\right) d s+K * \tilde{\rho}_{s}\left(X_{s}\right) \nabla f\left(s, X_{s}\right)\right] h\left(\tau, X_{0}\right) \mathbf{1}_{\{s \geq \tau\}} d s
\end{aligned}
$$

are seen to have vanishing expectation for all $f \in \mathcal{C}_{b}^{1,2}$. We take that expectation and use Fubini's theorem, and we conclude by Remark 2.5 and the definition of $\tilde{\rho}$.

Remark 2.8. By a standard argument using the semi-martingale decomposition of the coordinate processes $X^{i}$ and their products $X^{i} X^{j}$, we obtain that for $f \in$ $C_{b}^{1,2}$, the martingale part of $f\left(t, X_{t}\right)$ in (MP) is given by the stochastic integral $\sqrt{2 \nu} \int_{0}^{t} \nabla f\left(s, X_{s}\right) \mathbf{1}_{\{s \geq \tau\}} d B_{s}$, with respect to a Brownian motion $B$ defined on some extension of the canonical space.

Consequently, on the random interval $[0, \tau]$, the martingales in (MP) are null and $X_{t}=X_{0}$.

It follows that the second condition in (MP) is equivalent to the fact that

$$
\begin{aligned}
f\left(t, X_{t}\right) & -f\left(0, X_{0}\right)-\int_{0}^{t} \frac{\partial f}{\partial s}\left(s, X_{s}\right) d s \\
& -\int_{0}^{t}\left[\nu \triangle f\left(s, X_{s}\right)+K * \tilde{\rho}_{s}\left(X_{s}\right) \nabla f\left(s, X_{s}\right)\right] \mathbf{1}_{\{s \geq \tau\}} d s
\end{aligned}
$$

is a continuous $P$-martingale with respect to $\left(\mathcal{F}_{t}\right)$ for all $f \in C_{b}^{1,2}$.

\section{The PARTICLE SYSTEM}

In a first stage we deal with a regularized version of the kernel $K$. Let $\varphi: \mathbb{R}^{2} \rightarrow \mathbb{R}$ be a bounded and smooth function with bounded derivatives with $\|\varphi\|_{1}=1$. For $\varepsilon>0$ define

$$
K_{\varepsilon}=K * \varphi_{\varepsilon},
$$

where $\varphi_{\varepsilon}(x)=\frac{1}{\varepsilon^{2}} \varphi\left(\frac{x}{\varepsilon}\right)$. The function $¡ K_{\varepsilon}$ is bounded and smooth, and has bounded derivatives. We denote by $M_{\varepsilon}$ its sup-norm on $\mathbb{R}^{2}$ and by $L_{\varepsilon}$ a Lipschitz constant, that respectively behave like $\frac{1}{\varepsilon^{2}}$ and $\frac{1}{\varepsilon^{3}}$ when $\varepsilon<<1$. Notice that $\operatorname{div} K_{\varepsilon}=$ $(\operatorname{div} K) * \varphi_{\varepsilon}=0$.

In this section we fix $\varepsilon>0$, and consider a "mollified" version of equation (2):

$$
\frac{\partial v}{\partial t}+\left(K_{\varepsilon} * v \cdot \nabla\right) v=\nu \Delta v+g .
$$

We will adapt the usual McKean-Vlasov approach to give a probabilistic interpretation to (10) and construct an approximating stochastic particle system.

3.1. The nonlinear mollified process. Consider on some given probability space a 2-dimensional Brownian motion $B$ and a $\mathbb{R}_{+} \times \mathbb{R}^{2}$ valued random variable $\left(\tau, X_{0}\right)$ independent of $B$ with law $P_{0}$.

Theorem 3.1. There is existence and uniqueness, trajectorial and in law, for the following nonlinear stochastic differential equation in the sense of McKean:

$$
X_{t}^{\varepsilon}=X_{0}+\sqrt{2 \nu} \int_{0}^{t} \mathbf{1}_{s \geq \tau} d B_{s}+\int_{0}^{t} K_{\varepsilon} * \tilde{P}_{s}^{\varepsilon}\left(X_{s}^{\varepsilon}\right) \mathbf{1}_{s \geq \tau} d s, \quad t>0,
$$


under the conditions $\operatorname{law}\left(\tau, X_{0}\right)=P_{0}$ and $\operatorname{law}\left(\tau, X^{\varepsilon}\right)=P^{\varepsilon}$.

Proof. The proof is easily adapted from Theorem 1.1 in [21. Denote by $d_{T}$ the Kantorovich-Wasserstein distance on $\mathcal{C}_{T}$

$$
\begin{array}{r}
d_{T}\left(Q^{1}, Q^{2}\right):=\inf \left\{\int_{\left(\mathcal{C}_{T}\right)^{2}}\left[\sup _{t \in[0, T]}(|x(t)-y(t)| \wedge 1)+|\alpha-\beta|\right] \Pi(d \alpha, d x, d \beta, d y):\right. \\
\left.\Pi \text { has marginal laws } Q^{1} \text { and } Q^{2}\right\},
\end{array}
$$

and by $\mathcal{C}_{T}^{0}$ the closed subspace $\mathcal{C}_{T}^{0}=\left\{Q \in \mathcal{P}\left(\mathcal{C}_{T}\right): Q \circ\left(\tau, X_{0}\right)^{-1}=P_{0}\right\}$. Define a mapping $\Theta: \mathcal{C}_{T}^{0} \rightarrow \mathcal{C}_{T}^{0}$ associating with $Q$ the law $\Theta(Q)$ of the unique solution of

$$
X_{t}^{Q}=X_{0}+\sqrt{2 \nu} \int_{0}^{t} \mathbf{1}_{s \geq \tau} d B_{s}+\int_{0}^{t} K_{\varepsilon} * \tilde{Q}_{s}\left(X_{s}^{Q}\right) \mathbf{1}_{s \geq \tau} d s
$$

By trajectorial considerations, one can show that for each $t \leq T$,

$$
d_{t}\left(\Theta\left(Q^{1}\right), \Theta\left(Q^{2}\right)\right) \leq C(T) \int_{0}^{t} d_{s}\left(Q^{1}, Q^{2}\right) d s
$$

(with $d_{t}\left(Q^{1}, Q^{2}\right)$ the distance between the projections of $Q^{1}$ and $Q^{2}$ to $\mathcal{C}_{t}$ ). We deduce the existence of a unique fixed point for $\Theta$ and hence a unique solution in law. The trajectorial statement then follows from the Lipschitz property of $K_{\varepsilon}$ (see 21] for details).

We shall need in the sequel the stochastic flow associated with the nonlinear process (11)

$$
\xi_{s, t}^{\varepsilon}(x)=x+\sqrt{2 \nu}\left(B_{t}-B_{s}\right)+\int_{s}^{t} K_{\varepsilon} * \tilde{P}_{r}^{\varepsilon}\left(\xi_{s, r}^{\varepsilon}(x)\right) d r, \quad x \in \mathbb{R}^{2}, 0 \leq s \leq t .
$$

The regularity properties of $K_{\varepsilon} * \tilde{P}_{s}^{\varepsilon}(x)$ imply the existence of a continuous version $(s, t, x) \mapsto \xi_{s, t}^{\varepsilon}(x)$ such that $x \mapsto \xi_{s, t}^{\varepsilon}(x)$ is continuously differentiable for all $(s, t)$ (cf. Kunita [15]). Since $X_{t}^{\varepsilon}=X_{0}$ for all $t \leq \tau$, we have that

$$
X_{t}^{\varepsilon}=\xi_{\tau, t}^{\varepsilon}\left(X_{0}\right) \mathbf{1}_{\{t \geq \tau\}}+X_{0} \mathbf{1}_{\{t<\tau\}}
$$

Denote by

$$
G^{\varepsilon}(s, x ; t, y), \quad(s, x, t, y) \in\left(\mathbb{R}_{+} \times \mathbb{R}^{2}\right)^{2}, s<t,
$$

the density of $\xi_{s, t}^{\varepsilon}(x)$ (which is a continuous function of $(s, x, t, y)$; see [1]). Conditioning with respect to $\left(\tau, X_{0}\right)$, we obtain for bounded functions $f$ that

$$
\begin{aligned}
E\left(f\left(X_{t}^{\varepsilon}\right)\right)= & E\left[f\left(\xi_{\tau, t}^{\varepsilon}\left(X_{\tau}\right)\right) \mathbf{1}_{\{t \geq \tau\}}\right]+E\left[f\left(X_{0}\right) \mathbf{1}_{\{t<\tau\}}\right] \\
= & \int_{0}^{t} \int_{\left(\mathbb{R}^{2}\right)^{2}} f(y) G^{\varepsilon}(s, x ; y, t) d y P_{0}(d s, d x)+\int_{t}^{T} \int_{\mathbb{R}^{2}} f(x) P_{0}(d s, d x) \\
= & \int_{\mathbb{R}^{2}} f(x) \bar{w}_{0}(x) d x+\int_{0}^{t} \int_{\mathbb{R}^{2}}\left[\int_{\mathbb{R}^{2}} f(y) G^{\varepsilon}(s, x ; t, y) d y\right] \bar{g}_{s}(x) d x d s \\
& +\int_{t}^{T} \int_{\mathbb{R}^{2}} f(x) \bar{g}_{s}(x) d x d s
\end{aligned}
$$

where the notation $\bar{w}_{0}$ and $\bar{g}_{s}$ have been introduced in (6) . 
We deduce that for each $t \in] 0, T], X_{t}^{\varepsilon}$ has a bi-measurable density $\rho_{t}^{\varepsilon}(y)$. Similarly, we have

$$
\begin{aligned}
\int_{\mathbb{R}^{2}} f(x) \tilde{P}_{t}^{\varepsilon}(d x)= & E\left[f\left(\xi_{\tau, t}^{\varepsilon}\left(X_{0}\right)\right) h\left(\tau, X_{0}\right) \mathbf{1}_{\{\tau \leq t\}}\right]+E\left[f\left(X_{0}\right) h\left(\tau, X_{0}\right) \mathbf{1}_{\{\tau>t\}}\right] \\
= & \int_{\mathbb{R}^{2}} f(x) w_{0}(x) d x+\int_{0}^{t} \int_{\mathbb{R}^{2}}\left[\int_{\mathbb{R}^{2}} f(y) G^{\varepsilon}(s, x ; t, y) d y\right] g_{s}(x) d x d s \\
& +\int_{t}^{T} \int_{\mathbb{R}^{2}} f(x) g_{s}(x) d x d s
\end{aligned}
$$

and then $\tilde{P}_{t}^{\varepsilon}(d y)$ has a bi-measurable density $\tilde{\rho}_{t}^{\varepsilon}(y)$.

Remark 3.2. By construction,

$$
\sup _{\varepsilon>0} \sup _{t \in[0, T]}\left\|\tilde{\rho}_{t}^{\varepsilon}\right\|_{1} \leq\left\|w_{0}\right\|_{1}+\|g\|_{1, T} .
$$

Corollary 3.3. The function $\tilde{\rho}^{\varepsilon}$ is the unique weak solution of equation (10) in $L^{\infty}\left([0, T], L^{1}\right)$.

Proof. Write Itô's formula for $\phi\left(t, X_{t}^{\varepsilon}\right)$ and proceed as in Lemma 2.7 (the boundedness of $K_{\varepsilon}$ provides the required integrability condition). We obtain that $\tilde{\rho}_{t}^{\varepsilon} \in$ $L^{\infty}\left([0, T], L^{1}\right)$ solves

$$
\begin{aligned}
\int_{\mathbb{R}^{2}} \phi & (t, x) \tilde{\rho}_{t}^{\varepsilon}(x) d x=\int_{\mathbb{R}^{2}} \phi(0, x) w_{0}(x) d x+\int_{0}^{t} \int_{\mathbb{R}^{2}} \phi(s, x) g_{s}(x) d x d s \\
& +\int_{0}^{t} \int_{\mathbb{R}^{2}}\left[\frac{\partial \phi(s, x)}{\partial s}+\nu \triangle \phi(s, x)+K_{\varepsilon} * \tilde{\rho}_{s}^{\varepsilon}(x) \nabla \phi(s, x)\right] \tilde{\rho}_{s}^{\varepsilon}(x) d x d s
\end{aligned}
$$

for all $\phi \in C_{b}^{1,2}\left([0, T] \times \mathbb{R}^{2}\right)$. Using the boundedness of $K_{\varepsilon}$ to proceed as in Remark 2.4. we check next that $\tilde{\rho}^{\varepsilon}$ is a solution in $L^{\infty}\left([0, T], L^{1}\right)$ of the mollified mild equation

$$
w_{t}^{\varepsilon}(x)=G_{t}^{\nu} * w_{0}(x)+\int_{0}^{t} G_{t-s}^{\nu} * g_{s}(x) d s+\int_{0}^{t} \nabla G_{t-s}^{\nu} *\left[\left(K_{\varepsilon} * w_{s}^{\varepsilon}\right) w_{s}^{\varepsilon}\right](x) d s, \quad \varepsilon \geq 0 .
$$

If $v$ is another solution of (15), from Lemma 2.2 with $l=\infty, m=1$ we get

$$
\left\|\tilde{\rho}_{t}^{\varepsilon}-v_{t}\right\|_{1} \leq C(\varepsilon) \int_{0}^{t}(t-s)^{-\frac{1}{2}}\left\|\tilde{\rho}_{s}^{\varepsilon}-v_{s}\right\|_{1} d s
$$

and conclude uniqueness using Lemma 1.1

3.2. Stochastic particle approximations. We now define an interacting particle system which is naturally associated with the nonlinear process studied above. The system takes into account the random space-time births. Its pathwise existence and uniqueness can be proved by adapting standard arguments.

Definition 3.4. Consider a sequence $\left(B^{i}\right)_{i \in \mathbb{N}}$ of independent Brownian motions on $\mathbb{R}^{2}$ and a sequence of independent variables $\left(\tau^{i}, X_{0}^{i}\right)_{i \in \mathbb{N}}$ with values in $[0, T] \times \mathbb{R}^{2}$ distributed according to $P_{0}$, and independent of the Brownian motions. For a fixed 
$\varepsilon>0$, for each $n \in \mathbb{N}^{*}$, let us consider the interacting processes defined for $1 \leq i \leq n$ by

$$
X_{t}^{i n, \varepsilon}=X_{0}^{i}+\sqrt{2 \nu} \int_{0}^{t} \mathbf{1}_{\left\{s \geq \tau^{i}\right\}} d B_{s}^{i}+\int_{0}^{t} \mathbf{1}_{\left\{s \geq \tau^{i}\right\}} K_{\varepsilon} * \tilde{\mu}_{s}^{n, \varepsilon}\left(X_{s}^{i n, \varepsilon}\right) d s
$$

where

$$
\tilde{\mu}_{s}^{n, \varepsilon}=\frac{1}{n} \sum_{j=1}^{n} h\left(\tau^{j}, X_{0}^{j}\right) \mathbf{1}_{\left\{s \geq \tau^{j}\right\}} \delta_{X_{s}^{j n, \varepsilon}}
$$

is the weighted empirical measure of the system at time $s$ and

$$
K_{\varepsilon} * \tilde{\mu}_{s}^{n, \varepsilon}(z)=\frac{1}{n} \sum_{j=1}^{n} h\left(\tau^{j}, X_{0}^{j}\right) \mathbf{1}_{\left\{s \geq \tau^{j}\right\}} K_{\varepsilon}\left(z-X_{s}^{j n, \varepsilon}\right) .
$$

Hence, particles either have birth at time 0 or at a random time, and evolve thereafter as diffusive particles that interact following a mean field depending on the parameter $\varepsilon$. We introduce a coupling between these interacting processes and some independent copies of the limiting process defined in (11):

Definition 3.5. For $i \geq 1$, we define $\bar{X}^{i, \varepsilon}$ by

$$
\bar{X}_{t}^{i, \varepsilon}=X_{0}^{i}+\sqrt{2 \nu} \int_{0}^{t} \mathbf{1}_{\left\{s \geq \tau^{i}\right\}} d B_{s}^{i}+\int_{0}^{t} \mathbf{1}_{\left\{s \geq \tau^{i}\right\}} K_{\varepsilon} * \tilde{\rho}_{s}^{\varepsilon}\left(\bar{X}_{s}^{i, \varepsilon}\right) d s .
$$

By an adaptation of the proof of Proposition 2.2 in [13, we can show the following proposition.

Proposition 3.6. There exist positive constants $C_{1}, C_{2}$ such that for all $n \in \mathbb{N}$, $1 \leq i \leq n$ and $\varepsilon \in] 0,1[$,

$$
E\left(\sup _{t \leq T}\left|X_{t}^{i n, \varepsilon}-\bar{X}_{t}^{i, \varepsilon}\right|\right) \leq \frac{C_{1} \varepsilon}{\sqrt{n}} \exp \left(C_{2}\left(\left\|w_{0}\right\|_{1}+\|g\|_{1, T}\right)\left(\varepsilon^{-2}\right) T\right) .
$$

Remark 3.7. The function $h$ being bounded, is is not hard to deduce from the previous theorem that for all continuous bounded $f: \mathbb{R}^{2} \rightarrow \mathbb{R}$ and $\varepsilon>0$,

$$
E\left|\left\langle\tilde{\mu}_{t}^{n, \varepsilon}, f\right\rangle-\int_{\mathbb{R}^{2}} f(x) \tilde{\rho}_{t}^{\varepsilon}(x) d x\right| \rightarrow 0 \quad \text { when } n \rightarrow \infty .
$$

3.3. Density estimates. Due to the bad behavior of $K$ in the space $L^{1}$, it is necessary to obtain additional $L^{p}$ estimates for some $p>1$ in order to study the convergence of our scheme when $\varepsilon \rightarrow 0$. We present an argument based on the properties of the stochastic flow, and on a "stochastic version" of Liouville's theorem. In [6], Busnello also relied on the stochastic flow to obtain uniform in time estimates for some solutions to the vortex equation, but for the case without external field and under regularity of the initial condition.

Lemma 3.8. Let $J \xi_{s, t}^{\varepsilon}=\operatorname{det}\left(\nabla_{x} \xi_{s, t}^{\varepsilon}\right)$ be the Jacobian of the function $\xi_{s, t}^{\varepsilon}: \mathbb{R}^{2} \rightarrow \mathbb{R}^{2}$. Then

$$
J \xi_{s, t}^{\varepsilon}(x)=1+\int_{s}^{t} \operatorname{div}\left[K_{\varepsilon} * \tilde{P}_{r}^{\varepsilon}\left(\xi_{s, r}^{\varepsilon}(x)\right)\right] J \xi_{s, r}^{\varepsilon} d r .
$$

Since div $K_{\varepsilon} * \tilde{P}_{r}^{\varepsilon}\left(\xi_{s, r}^{\varepsilon}(x)\right)=0$ we have that $J \xi_{s, t}^{\varepsilon}(x)=1$ for all $(s, t, x)$.

The proof is similar as the classic version (e.g. [8] Ch. 1), since the diffusion coefficient in (12) is constant. 
Lemma 3.9. Let $\rho^{\varepsilon}$ and $\tilde{\rho}^{\varepsilon}$ be, respectively, the family of densities of $X$ under $P^{\varepsilon}$ and the family of weighted densities associated with $P^{\varepsilon}$ through (8). Let $p \in[1, \infty]$ and assume that $w_{0} \in L^{p}$ and $g \in L^{1}\left([0, T], L^{p}\right)$. Then, for all $\varepsilon>0$ and $t \in[0, T]$, we have

$$
\begin{aligned}
\text { i) }\left\|\tilde{\rho}_{t}^{\varepsilon}\right\|_{p} & \leq\left\|w_{0}\right\|_{p}+\int_{0}^{t}\left\|g_{s}\right\|_{p} d s, \\
\text { ii) }\left\|\rho_{t}^{\varepsilon}\right\|_{p} & \leq \frac{\left\|w_{0}\right\|_{p}+\int_{0}^{T}\left\|g_{s}\right\|_{p} d s}{\left\|w_{0}\right\|_{1}+\int_{0}^{T}\left\|g_{s}\right\|_{1} d s} .
\end{aligned}
$$

Proof. Consider a fixed function $\psi \in \mathcal{D}$ and $t>0$. By the Feynman-Kac formula, the function $\phi^{t}(s, x):=E\left(\psi\left(\xi_{s, t}^{\varepsilon}(x)\right)\right)$ is the unique solution of the Cauchy problem

$$
\begin{aligned}
\frac{\partial \phi(s, x)}{\partial s}+\nu \triangle \phi(s, x)+K_{\varepsilon} * \tilde{\rho}_{s}^{\varepsilon}(x) \nabla \phi(s, x) & =0 \quad \text { for all }(s, x) \in\left[0, t\left[\times \mathbb{R}^{2},\right.\right. \\
\phi(t, x) & =\psi .
\end{aligned}
$$

Replacing $\phi^{t}$ in the weak equation (14) and using Fubini's theorem, we obtain

$$
\begin{aligned}
\int_{\mathbb{R}^{2}} \psi(x) \tilde{\rho}_{t}^{\varepsilon}(x) d x= & \int_{\mathbb{R}^{2}} \phi^{t}(0, x) w_{0}(x) d x+\int_{0}^{t} \int_{\mathbb{R}^{2}} \phi^{t}(s, x) g_{s}(x) d x d s \\
= & E\left(\int_{\mathbb{R}^{2}}\left[\psi\left(\xi_{0, t}^{\varepsilon}(x)\right) w_{0}(x)\right] d x\right) \\
& +\int_{0}^{t} E\left(\int_{\mathbb{R}^{2}}\left[\psi\left(\xi_{s, t}^{\varepsilon}(x)\right) g_{s}(x)\right] d x\right) d s
\end{aligned}
$$

and so

$$
\left|\int_{\mathbb{R}^{2}} \psi(x) \tilde{\rho}_{t}^{\varepsilon}(x) d x\right| \leq E\left[\left\|\psi\left(\xi_{0, t}^{\varepsilon}(\cdot)\right)\right\|_{p^{*}}\right]\left\|w_{0}\right\|_{p}+\int_{0}^{t} E\left[\left\|\psi\left(\xi_{s, t}^{\varepsilon}(\cdot)\right)\right\|_{p^{*}}\right]\left\|g_{s}\right\|_{p} d s .
$$

Thanks to Lemma 3.8, we conclude that

$$
\left|\int_{\mathbb{R}^{2}} \psi(x) \tilde{\rho}_{t}^{\varepsilon}(x) d x\right| \leq\|\psi\|_{p^{*}}\left(\left\|w_{0}\right\|_{p}+\int_{0}^{t}\left\|g_{s}\right\|_{p} d s\right)
$$

which proves $i$ ). To prove $i$ i), define a sub-probability density $\hat{\rho}_{t}^{\varepsilon}$ by $\int_{\mathbb{R}^{2}} \psi(x) \hat{\rho}_{t}^{\varepsilon}(x) d x$ $=E\left(\psi\left(X_{t}^{\varepsilon}\right) \mathbf{1}_{\{t \geq \tau\}}\right)$. Writing Itô's formula for $f\left(t, X_{t}^{\varepsilon}\right)$, multiplying by $\mathbf{1}_{\{t \geq \tau\}}$ and taking expectations, we check that

$$
\begin{aligned}
& \int_{\mathbb{R}^{2}} \phi_{t}(x) \hat{\rho}_{t}^{\varepsilon}(x) d x=\int_{\mathbb{R}^{2}} \phi(0, x) \bar{w}_{0}(x) d x+\int_{0}^{t} \int_{\mathbb{R}^{2}} \phi(s, x) \bar{g}_{s}(x) d x d s \\
&+\int_{0}^{t} \int_{\mathbb{R}^{2}}\left[\frac{\partial \phi(s, x)}{\partial s}+\nu \triangle \phi(s, x)+K_{\varepsilon} * \tilde{\rho}_{s}^{\varepsilon}(x) \nabla \phi(s, x)\right] \hat{\rho}_{s}^{\varepsilon}(x) d x d s .
\end{aligned}
$$

We deduce as previously that

$$
\left|\int_{\mathbb{R}^{2}} \psi(x) \hat{\rho}_{t}^{\varepsilon}(x) d x\right| \leq \frac{\|\psi\|_{p^{*}}}{\left\|w_{0}\right\|_{1}+\|g\|_{1, T}}\left(\left\|w_{0}\right\|_{p}+\int_{0}^{t}\left\|g_{s}\right\|_{p} d s\right) .
$$

The desired estimate for $\rho_{t}^{\varepsilon}$ follows from here, since

$$
E\left(\psi\left(X_{t}^{\varepsilon}\right) \mathbf{1}_{\{t<\tau\}}\right)=E\left(\psi\left(X_{0}\right) \mathbf{1}_{\{t<\tau\}}\right)=\int_{t}^{T} \int_{\mathbb{R}^{2}} \psi(x) \frac{\left|g_{s}(x)\right|}{\left\|w_{0}\right\|_{1}+\|g\|_{1, T}} d x d s .
$$




\section{4. $L^{1} \cap L^{p}$ DATA: EXISTENCE, UNIQUENESS AND PATHWISE APPROXIMATION}

The main goal of this section is to construct the pathwise unique nonlinear process related to the true Biot-Savart kernel, as a limit of the mollified nonlinear processes, when $\varepsilon \rightarrow 0$. We shall need fundamental continuity properties of the operator $f \mapsto K * f$ and regularity estimates on the solution of the vortex equation.

Lemma 4.1. Let $p \in(1,2)$ and $\frac{1}{q}=\frac{1}{p}-\frac{1}{2}$. For each $f \in L^{p}$ and $x \in \mathbb{R}^{2}$ the integral $K * f(x)$ is absolutely convergent. Furthermore, there is a constant $C_{p, q}>0$ such that

$$
\|K * f\|_{q} \leq C_{p, q}\|f\|_{p} \quad \text { for all } \in f \in L^{p} .
$$

ii)

$$
\|K * f\|_{W^{1, q}} \leq C_{p, q}\|f\|_{W^{1, p}} \quad \text { for all } \in f \in W^{1, p} .
$$

Proof. The absolute convergence of $K * f(x)$ and statement i) follow from the analogous results for the Riesz transform

$$
f \in L^{p} \mapsto \int_{\mathbb{R}^{2}} \frac{f(y)}{|x-y|} d y \in L^{q}(d x)
$$

(cf. Theorem 1, Ch. 5, in Stein [20]). To prove ii), using i) we need only to check that $K *$ commutes with derivatives on $\mathcal{D}$. This is easy by dominated convergence.

Remark 4.2. Lemma 4.1 with the same constants applies to each mollified kernel $K_{\varepsilon}$.

We now introduce the adequate spaces to work in. For measurable $w:[0, T] \times$ $\mathbb{R}^{2} \rightarrow \mathbb{R}$ and real numbers $p \in[1, \infty]$ and $r \geq p$ we introduce the norms

- $\|w\|_{0, p, T}=\sup _{0 \leq t \leq T}\left\|w_{t}\right\|_{p}$

- $\|w\|_{0, r,(T ; p)}=\sup _{0 \leq t \leq T}\left\{t^{\frac{1}{p}-\frac{1}{r}}\left\|w_{t}\right\|_{r}\right\}$,

and we denote the associated Banach spaces, respectively, by

$$
F_{0, p, T} \text { and } F_{0, r,(T ; p)} \text {. }
$$

We write $K_{0}=K$, and for each $\varepsilon \geq 0$, we define the bilinear operator $\mathcal{B}^{\varepsilon}$ on measurable functions $v, w:[0, T] \times \mathbb{R}^{2} \rightarrow \mathbb{R}$, by

$$
\mathcal{B}^{\varepsilon}(v, w)(t, x)=\int_{0}^{t} \int_{\mathbb{R}^{2}} \nabla G_{t-s}^{\nu}(x-y) \cdot K_{\varepsilon} * v_{s}(y) w_{s}(y) d y d s .
$$

Accordingly, we also write $\mathcal{B}=\mathcal{B}^{0}$.

We denote by $W_{0}$ the function

$$
W_{0}(t, x)=G_{t}^{\nu} * w_{0}(x)+\int_{0}^{t} G_{t-s}^{\nu} * g_{s}(x) d s .
$$

Lemma 4.3. $\quad$ i) Let $p \in[1, \infty]$ and assume $w_{0} \in L^{p}$ and $g \in F_{0, p, T}$. Then, we have

$$
W_{0} \in F_{0, r,(T ; p)} \quad \text { for all } r \geq p \text {. }
$$


ii) For each $r \geq \frac{4}{3}, v, w \in F_{0, r, T}$, and each $t \leq T$, we have

$$
\sup _{\varepsilon \geq 0}\left\|K_{\varepsilon} * v(t) w(t)\right\|_{\frac{2 r}{4-r}} \leq C\|v(t)\|_{r}\|w(t)\|_{r} .
$$

iii) If $\frac{4}{3} \leq p<2, p \leq r<2$ and $\frac{2 r}{4-r} \leq r^{\prime}<\frac{r}{2-r}$, then $\mathcal{B}^{\varepsilon}:\left(F_{0, r,(T ; p)}\right)^{2} \rightarrow$ $F_{0, r^{\prime},(T ; p)}$ is well defined for each $\varepsilon \geq 0$, and for all $v, w \in F_{0, r,(T ; p)}$,

$$
\sup _{\varepsilon \geq 0}\left\|\mathcal{B}^{\varepsilon}(v, w)\right\|_{0, r^{\prime},(T ; p)} \leq C T^{1-\frac{1}{p}}\|v\|_{0, r,(T ; p)}\|w\|_{0, r,(T ; p)} .
$$

iv) If $\frac{4}{3} \leq p<2, w_{0} \in L^{p}$ and $g \in F_{0, p, T}$, we have for all $r \in\left[p, \frac{2 p}{2-p}\right]$

$$
\sup _{\varepsilon \geq 0}\left\|\tilde{\rho}^{\varepsilon}\right\|_{0, r,(T ; p)}<\infty .
$$

Proof. Part i) follows from Lemma 2.2, and the estimate

$$
\left\|\int_{0}^{t} G_{t-s}^{\nu} * g_{s} d s\right\|_{r} \leq C(p, r) t^{1+\frac{1}{r}-\frac{1}{p}}\left(\sup _{t \in[0, T]}\left\|g_{t}\right\|_{p}\right)
$$

for some constant $C(p, r)>0$ since $1 / p<1 / r+1$.

ii) Notice that $1 \leq \frac{2 r}{4-r}$. Equation (23) is immediately obtained from Lemma 4.1. Remark 4.2 and Hölder's inequality.

iii) By (23), noticing that $1 \leq \frac{2 r}{4-r} \leq r^{\prime}$ and by Lemma 2.2 and Lemma 4.1 , we have

$$
\begin{aligned}
\left\|\mathcal{B}^{\varepsilon}(v, w)_{t}\right\|_{r^{\prime}} & \leq C \int_{0}^{t}(t-s)^{\frac{1}{r^{\prime}}-\frac{2}{r}}\left\|v_{s}\right\|_{r}\left\|w_{s}\right\|_{r} d s \\
& \leq C\|v\|_{0, r,(T ; p)}\|w\|_{0, r,(T ; p)} \int_{0}^{t}(t-s)^{\frac{1}{r^{\prime}}-\frac{2}{r}} s^{\frac{2}{r}-\frac{2}{p}} d s \\
& =C t^{1+\frac{1}{r^{\prime}}-\frac{2}{p}}\|v\|_{0, r,(T ; p)}\|w\|_{0, r,(T ; p)}
\end{aligned}
$$

with constants that do not depend on $\varepsilon \geq 0$. In the last step we have used the fact that $\frac{1}{r}-\frac{1}{p}>-\frac{1}{2}$ because $r<2<\frac{2 p}{2-p}$. The statement follows.

iv) By Lemma 3.9, we have

$$
\sup _{\varepsilon \geq 0}\left\|\tilde{\rho}^{\varepsilon}\right\|_{0, p, T}<\infty .
$$

Observe that $\frac{p}{2-p} \geq 2$. We define $p_{1}:=\frac{4 p}{2+p} \in(p, 2)$ and apply iii) to $r=p$ and $r^{\prime}=p_{1}$ which yields $\sup _{\varepsilon \geq 0}\left\|\tilde{\rho}^{\varepsilon}\right\|_{0, p_{1},(T ; p)}<\infty$, considering equation (15) and i). We now apply iii) to $r=p_{1}$ and some $r^{\prime} \in\left[\frac{2 p_{1}}{4-p_{1}}, \frac{p_{1}}{2-p_{1}}\right)=\left[p, \frac{2 p}{2-p}\right)$ and conclude in a similar way.

To get the conclusion for the limiting value $\frac{2 p}{2-p}$ we observe that for $\varepsilon>0$ small enough, we can now apply the previous arguments, first to $r=p+\varepsilon \in[p, 2)$ and $r^{\prime}=p_{2}:=\frac{4(p+\varepsilon)}{2+p+\varepsilon} \in(p, 2)$, and then to $r=p_{2}$ and $r^{\prime}=\frac{2 p}{2-p} \in\left[p+\varepsilon, \frac{2(p+\varepsilon)}{2-(p+\varepsilon)}\right)$.

In the sequel we shall make the following type of assumption on the data:

$$
\left(\mathbf{H}_{\mathbf{p}}\right) \text { : }
$$

$$
w_{0} \in L^{p}\left(\mathbb{R}^{2}\right) \text { and } g \in F_{0, p, T} .
$$

In view of the continuity property of the Biot-Savart operator, and of part iii) of the previous lemma, we will always consider

$$
p=1 \text { or } p \in\left[\frac{4}{3}, 2\right) \text {. }
$$


4.1. Convergence of the mollified solutions for $L^{1} \cap L^{p}$ data. For technical reasons, we will make a particular choice of approximating kernels $K_{\varepsilon}(x)=K *$ $\varphi_{\varepsilon}(x)$. We take $\varphi$ as the cutoff function with radial symmetry given by Raviart [19] in a general context of approximations, and proposed by Bossy [3] for a numerical study of the vortex algorithm:

$$
\varphi(x)=\frac{2\left(2-r^{2}\right)}{\pi\left(1+r^{2}\right)^{4}}, \quad r=|x| .
$$

The following is proved in [18].

Lemma 4.4. For each $l \in[1,2)$ and a positive constant $C$ depending only on $l$, we have

$$
\left\|K_{\varepsilon}-K\right\|_{l} \leq C \varepsilon^{\frac{2-l}{l}} .
$$

Proposition 4.5. Assume that $\left(\mathbf{H}_{\mathbf{p}}\right)$ holds, with $p \in\left[\frac{4}{3}, 2\right)$. There exists a solution $w \in F_{0, p, T}$, the mild vortex equation (5). Moreover, for any $l \in[1,2)$ and $\varepsilon>0$ it holds that

$$
\sup _{t \leq T}\left\|\tilde{\rho}_{t}^{\varepsilon}-w_{t}\right\|_{p} \leq C_{l} \varepsilon^{\frac{2-l}{l}}
$$

Proof. By Lemma 4.4, for $1 \geq l<2$ and $\varepsilon>\varepsilon^{\prime}>0$ we have $\left\|K_{\varepsilon}-K_{\varepsilon^{\prime}}\right\|_{l} \leq C \varepsilon^{\frac{2-l}{l}}$. In view of (15), Lemma 2.2 and Lemma 4.3, we have, for $t \leq T$,

$$
\begin{aligned}
&\left\|\tilde{\rho}_{t}^{\varepsilon}-\tilde{\rho}_{t}^{\varepsilon^{\prime}}\right\|_{p} \leq \int_{0}^{t} \| \nabla G_{t-s}^{\nu} *\left[\left(K_{\varepsilon} * \tilde{\rho}_{s}^{\varepsilon}\right) \tilde{\rho}_{s}^{\varepsilon}-\left(K_{\varepsilon^{\prime}} * \tilde{\rho}_{s}^{\varepsilon^{\prime}}\right) \tilde{\rho}_{s}^{\varepsilon^{\prime}} \|_{p} d s\right. \\
& \leq C \int_{0}^{t}(t-s)^{-\frac{1}{p}}\left(\left\|\left(K_{\varepsilon} * \tilde{\rho}_{s}^{\varepsilon}\right) \tilde{\rho}_{s}^{\varepsilon}-\left(K_{\varepsilon^{\prime}} * \tilde{\rho}_{s}^{\varepsilon}\right) \tilde{\rho}_{s}^{\varepsilon}\right\|_{\frac{2 p}{4-p}}\right. \\
&+\left\|\left(K_{\varepsilon^{\prime}} * \tilde{\rho}_{s}^{\varepsilon}\right) \tilde{\rho}_{s}^{\varepsilon}-\left(K_{\varepsilon^{\prime}} * \tilde{\rho}_{s}^{\varepsilon^{\prime}}\right) \tilde{\rho}_{s}^{\varepsilon}\right\|_{\frac{2 p}{4-p}} \\
&\left.+\left\|\left(K_{\varepsilon^{\prime}} * \tilde{\rho}_{s}^{\varepsilon^{\prime}}\right)\left(\tilde{\rho}_{s}^{\varepsilon}-\tilde{\rho}_{s}^{\varepsilon^{\prime}}\right)\right\|_{\frac{2 p}{4-p}}\right) d s \\
& \leq C\left\|\tilde{\rho}^{\varepsilon}\right\|_{0, p, T}\left\|K_{\varepsilon^{\prime}}-K_{\varepsilon}\right\|_{l}\left\|\tilde{\rho}^{\varepsilon}\right\|_{0, r,(T ; p)} \int_{0}^{t}(t-s)^{-\frac{1}{p}} s^{\frac{1}{r}-\frac{1}{p}} d s \\
&+C\left(\left\|\tilde{\rho}^{\varepsilon}\right\|_{0, p, T}+\left\|\tilde{\rho}^{\varepsilon^{\prime}}\right\|_{0, p, T}\right) \int_{0}^{t}(t-s)^{-\frac{1}{p}}\left\|\tilde{\rho}_{s}^{\varepsilon}-\tilde{\rho}_{s}^{\varepsilon^{\prime}}\right\|_{p} d s,
\end{aligned}
$$

with $r \in\left(p, \frac{2 p}{2-p}\right]$ given by the relation $\frac{1}{p}-\frac{1}{2}=\frac{1}{r}+\frac{1}{l}-1$. The last inequality follows from Young's inequality for the first term and from (23) for the last two terms. Since $\frac{1}{r}-\frac{1}{p}+1>0$, Lemmas 4.3 iv) and Lemma 4.4 imply that

$$
\left\|\tilde{\rho}_{t}^{\varepsilon}-\tilde{\rho}_{t}^{\varepsilon^{\prime}}\right\|_{p} \leq C \sup _{\varepsilon}\left\|\tilde{\rho}^{\varepsilon}\right\|_{0, p, T}\left(\varepsilon^{\frac{2-l}{l}} \sup _{\varepsilon}\left\|\tilde{\rho}^{\varepsilon}\right\|_{0, r,(T ; p)}+C \int_{0}^{t}(t-s)^{-\frac{1}{p}}\left\|\tilde{\rho}_{s}^{\varepsilon}-\tilde{\rho}_{s}^{\varepsilon^{\prime}}\right\|_{p} d s\right)
$$

which together with Lemma 1.1 yields $\sup _{t \leq T}\left\|\tilde{\rho}_{t}^{\varepsilon}-\tilde{\rho}_{t}^{\varepsilon^{\prime}}\right\|_{p} \leq C \varepsilon^{\frac{2-l}{l}}$. The result follows.

Bringing together previous statements, we have the following result:

Theorem 4.6. Under $\left(\mathbf{H}_{\mathbf{1}}\right)$ and $\left(\mathbf{H}_{\mathbf{p}}\right)$ with $p \in\left[\frac{4}{3}, 2\right)$ the mild vortex equation with external force field (5) has a unique solution $w$ in the space $F_{0, p, T} \cap F_{0,1, T}$, which 
moreover satisfies

$$
\|w(t)\|_{p} \leq\left\|w_{0}\right\|_{p}+\int_{0}^{t}\left\|g_{s}\right\|_{p} d s .
$$

Proof. Thanks to Proposition 4.5] and Lemma 3.9, we only need to check uniqueness and the fact that $w \in F_{0,1, T}$. The latter is proved by iteratively using Lemma 4.3 iii); see Lemma 2.4 in [10] for details. By arguments used in Lemma 4.3 ii) with $p=r=r^{\prime}$, we also get $\left\|w_{t}-w_{t}^{\prime}\right\|_{p} \leq C \int_{0}^{t}(t-s)^{-\frac{1}{p}}\left\|w_{s}-w_{s}^{\prime}\right\|_{p} d s$ for two solutions $w, w^{\prime}$, and Lemma 1.1 yields uniqueness.

Remark 4.7. The statement of Theorem 4.6 also holds for equation (15). Hence, the unique solution $w^{\varepsilon} \in F_{0, p, T} \cap F_{0,1, T}\left(p \in\left[\frac{4}{3}, 2\right)\right)$ of (15) is given by $w^{\varepsilon}=\tilde{\rho}^{\varepsilon}$ if $\varepsilon>0$, or by $w^{0}=w$.

4.2. Regularity estimates. To show strong existence and uniqueness for the limiting process, and pathwise convergence of the mollified processes when $\varepsilon \rightarrow 0$, we need to prove some uniform (in $\varepsilon$ ) regularity properties for functions $\tilde{\rho}^{\varepsilon}$.

For $T>0$ and $r \geq p$ we introduce additional norms

$$
\begin{aligned}
& \text { - }\|v\|_{1, r,(T ; p)}=\sup _{0 \leq t \leq T}\left\{t^{\frac{1}{p}-\frac{1}{r}}\left\|v_{t}\right\|_{r}+t^{\frac{1}{2}+\left(\frac{1}{p}-\frac{1}{r}\right)}\left\|\nabla v_{t}\right\|_{r}\right\}, \\
& \text { - }\|v\|_{1, p, T}=\|v\|_{1, p,(T ; p)}=\sup _{0 \leq t \leq T}\left\{\left\|v_{t}\right\|_{p}+t^{\frac{1}{2}}\left\|\nabla v_{t}\right\|_{p}\right\},
\end{aligned}
$$

and the associated Banach spaces

$$
F_{1, r,(T ; p)} \quad \text { and } \quad F_{1, p, T} .
$$

Lemma 4.8. $\quad$ i) Assume $\left(\mathbf{H}_{\mathbf{p}}\right)$ with $\frac{4}{3} \leq p<2$. Then we have

$$
W_{0} \in F_{1, r,(T ; p)} \quad \text { for all } r \in\left[p, \frac{2 p}{2-p}\right) \text {. }
$$

ii) If $\frac{4}{3} \leq p<2, p \leq r<2$ and $\frac{2 r}{4-r} \leq r^{\prime}<\frac{r}{2-r}$, then $\mathcal{B}^{\varepsilon}:\left(F_{1, r,(T ; p)}\right)^{2} \rightarrow$ $F_{1, r^{\prime},(T ; p)}$ is well defined for each $\varepsilon \geq 0$ and

$$
\sup _{\varepsilon \geq 0}\left\|\mathcal{B}^{\varepsilon}(v, u)\right\|_{1, r^{\prime},(T ; p)} \leq C T^{1-\frac{1}{p}}\|v\|_{1, r,(T ; p)}\|u\|_{1, r,(T ; p)} .
$$

Proof. i) For $p \in[1,2)$ the function $t \mapsto t^{-\frac{1}{2}+\frac{1}{r}-\frac{1}{p}}$ is integrable in 0 if and only if $r<\frac{2 p}{2-p}$. Taking the gradient of $G_{t-s}^{\nu} * g_{s}$ under the time integral and using Lemma 2.2 we obtain

$$
\left\|\nabla\left(\int_{0}^{t} G_{t-s}^{\nu} * g_{s} d s\right)\right\|_{r} \leq C^{\prime}(p, r) t^{\frac{1}{2}+\frac{1}{r}-\frac{1}{p}}\left(\sup _{t \in[0, T]}\left\|g_{t}\right\|_{p}\right)
$$

for some constant $C^{\prime}(p, r)>0$, which implies that $W_{0} \in F_{1, r,(T ; p)}$.

ii) It is enough to check the properties for $\mathcal{B}$. If $v, u \in F_{1, r,(T ; p)}$, then $\left(K * v_{t}\right) u_{t} \in$ $W^{1, \frac{2 r}{4-r}}$, and integrating by parts yields

$$
\mathcal{B}(v, u)(t, x)=\int_{0}^{t} \int_{\mathbb{R}^{2}} G_{t-s}^{\nu}(x-y)\left(K * v_{s}\right)(y) \cdot \nabla u_{s}(y) d y d s .
$$

By adapting arguments of Proposition 3.1 in [10, we can take the derivatives in $x$ on both sides of the previous equation. Then, using the fact that $\frac{2}{r}-\frac{2}{p}-\frac{1}{2}>-1$, it is not hard to adapt the arguments of Lemma 4.3 iii) to get the required estimate for the derivatives. We just notice that the " $t$ - $\frac{1}{2}$-worst behavior at $t=0$ " of the 
derivative of $u$ is exactly compensated by the higher power of $t$ required in the derivative's part of the norm $\|\cdot\|_{1, r,(T ; p)}$.

Remark 4.9. By the previous lemma, and since $\frac{2 p}{4-p} \leq p<\frac{p}{2-p}$, equation (15) in the space $F_{1, p, T}$, with $\frac{4}{3} \leq p<2$, is equivalent to the abstract equation

$$
w^{\varepsilon}=W_{0}+\mathcal{B}^{\varepsilon}\left(w^{\varepsilon}, w^{\varepsilon}\right) .
$$

Proposition 4.10. Let $p \in\left[\frac{4}{3}, 2\right)$. Then, there is a constant $\lambda_{p}>0$ independent of $\varepsilon \geq 0$ such that for all $\theta>0$ and $w_{0} \in L^{p}, g \in F_{0, p, \theta}$ satisfying

$$
\theta^{1-\frac{1}{p}}\left(\left\|w_{0}\right\|_{p}+\theta\|g\|_{0, p, \theta}\right)<\lambda_{p},
$$

Equation (15) with $\varepsilon \geq 0$, has a unique solution in $F_{1, p, \theta}$ such that $\left\|w^{\varepsilon}\right\|_{1, p, \theta} \leq$ $2\left\|W_{0}\right\|_{1, p, \theta}$.

Proof. From Lemma 4.8 ii) (with $r=r^{\prime}=p^{\prime}=p$ ), the operators $\mathcal{B}^{\varepsilon}:\left(F_{1, p, \theta}\right)^{2} \rightarrow$ $F_{1, p, \theta}$ are continuous with norm bounded by $\theta^{1-\frac{1}{p}}$ times a constant $C(p)>0$ not depending on $\theta$ or $\varepsilon \geq 0$. Furthermore, from the proofs of Lemma 2.2 and Lemmas 4.3 i) and 4.8 i) there is $\tilde{C}(p)>0$ such that

$$
\left\|W_{0}\right\|_{1, p, \theta} \leq \tilde{C}(p)\left(\left\|w_{0}\right\|_{p}+\theta\|g\|_{0, p, \theta}\right) .
$$

Hence, by a standard fixed point argument for bilinear operators in Banach spaces (see Cannone [7, Ch. 1), the asserted solution $w^{\varepsilon} \in F_{1, p, \theta}$ to the abstract equation (31) exists as soon as

$$
4 C(p) \theta^{1-\frac{1}{p}}\left(\left\|w_{0}\right\|_{p}+\theta\|g\|_{0, p, \theta}\right) \tilde{C}(p)<1 .
$$

Theorem 4.11. Assume $\left(\mathbf{H}_{\mathbf{1}}\right)$ and $\left(\mathbf{H}_{\mathbf{p}}\right)$ with $p \in\left[\frac{4}{3}, 2\right)$ and let $w^{\varepsilon} \in F_{0, p, T} \cap F_{0,1, T}$, $\varepsilon \geq 0$, be the solution of (15).

i) We have

$$
\sup _{\varepsilon \geq 0}\left\|w^{\varepsilon}\right\|_{1, p, T}<\infty
$$

ii) For each $r \in\left[p, \frac{2 p}{2-p}\right)$

$$
\sup _{\varepsilon \geq 0}\left\|w^{\varepsilon}\right\|_{1, r,(T ; p)}<\infty .
$$

Proof. i) We follow the lines of Lemma 4.4 in 10 to deal with $w^{\varepsilon}$ at time 0 . By the semigroup property of $G_{t}^{\nu}$ and the estimates of Lemma 2.2, it is checked that

$$
w_{r+t}^{\varepsilon}(x)=W_{(r)}^{\varepsilon}(t, x)+\int_{0}^{t} \nabla G_{t-s}^{\nu} *\left[\left(K_{\varepsilon} * w_{r+s}^{\varepsilon}\right) w_{r+s}^{\varepsilon}\right](x) d s,
$$

for all $t \in(0, T-r]$, where $W_{(r)}^{\varepsilon}(t, x):=G_{t}^{\nu} * w_{r}^{\varepsilon}(x)+\int_{0}^{t} G_{t-s}^{\nu} * g_{s+r}(x) d s$. Then, as in the proof of Proposition 4.10 and with the same constant $\tilde{C}(p)$, we have

$$
\left\|W_{(r)}^{\varepsilon}\right\|_{1, p, \theta} \leq \tilde{C}(p)\left(\left\|w_{r}^{\varepsilon}\right\|_{p}+\theta\left\|g_{\cdot+r}\right\|_{0, p, \theta}\right) \leq \tilde{C}(p)\left(\left\|w_{0}\right\|_{p}+2 T\|g\|_{0, p, T}\right)
$$

for all $\theta \in[0, T-r]$, the last inequality owing to Lemma 3.9.

Now, thanks to Proposition 4.10 we can find some small enough $\theta_{0} \in[0, T]$ such that equation (32) has a solution, say $v_{(r)}^{\varepsilon}$, in the space $F_{1, p, \theta_{0}}$. By uniqueness in 
the space $F_{0, p, \theta_{0} \wedge(T-r)}$ holding for (32) and each $r$, we have that $w_{r+\cdot}^{\varepsilon}(\cdot)=v_{(r)}^{\varepsilon} \in$ $F_{1, p, \theta_{0} \wedge(T-r)}$, with

$$
\left\|w_{r+\cdot}^{\varepsilon}(\cdot)\right\|_{1, p, \theta_{0}} \leq 2\left\|W_{(r)}^{\varepsilon}\right\|_{1, p, \theta_{0}} .
$$

This of course implies that $w_{t}^{\varepsilon} \in W^{1, p}$ for strictly positive $t$.

Now choose $r_{k}:=k \frac{\theta_{0}}{2}$ so that $w_{r_{k}+t}^{\varepsilon}=w_{r_{k-1}+\frac{\theta_{0}}{2}+t}^{\varepsilon}$ for $t \in\left[0, \frac{\theta_{0}}{2}\right]$ and $k \in$ $\left\{1, \ldots,\left[\frac{2 T}{\theta_{0}}\right]\right\}$. For such $t$ and $k$, we have by (34) and (33) that

$$
\begin{aligned}
\left(r_{k}+t\right)^{\frac{1}{2}}\left\|\nabla w_{r_{k}+t}^{\varepsilon}\right\|_{p} & \leq \theta_{0}^{-\frac{1}{2}}\left(r_{k}+t\right)^{\frac{1}{2}}\left(t+\frac{\theta_{0}}{2}\right)^{\frac{1}{2}}\left\|\nabla w_{\left(r_{k-1}\right)+t+\frac{\theta_{0}}{2}}^{\varepsilon}\right\|_{p} \\
& \leq C\left(\frac{T}{\theta_{0}}\right)^{\frac{1}{2}}\left\|W_{\left(r_{k-1}\right)}\right\|_{1, p, \theta_{0}} \leq C\left(\frac{T}{\theta_{0}}\right)^{\frac{1}{2}}\left(\left\|w_{0}\right\|_{p}+2 T\|g\|_{0, p, T}\right) .
\end{aligned}
$$

This and (34) with $r=0$ yield assertion i). The proof of ii) is done in a similar way as in Lemma 4.3 iv), using Lemma 4.8 .

Corollary 4.12. Under the assumptions of Theorem 4.11, we have

i)

$$
\sup _{\varepsilon \geq 0} \sup _{t \in[0, T]}\left\{t^{\frac{1}{2}}\left(\left\|K_{\varepsilon} * w_{t}^{\varepsilon}\right\|_{\infty}+\left\|K_{\varepsilon} * w_{t}^{\varepsilon}\right\|_{\mathcal{C}^{2-\frac{2}{p}}}\right)\right\}<\infty
$$

ii) for all $r \in\left(2, \frac{2 p}{2-p}\right]$,

$$
\sup _{\varepsilon \geq 0} \sup _{t \in[0, T]}\left\{t^{\frac{1}{2}-\frac{1}{r}+\frac{1}{p}}\left(\left\|\nabla K_{\varepsilon} * w_{t}^{\varepsilon}\right\|_{\infty}+\left\|\nabla K_{\varepsilon} * w_{t}^{\varepsilon}\right\|_{\mathcal{C}^{1-\frac{2}{r}}}\right)\right\}<\infty .
$$

Here, $\|\cdot\|_{\mathcal{C}^{\alpha}}$ is the Hölder norm of index $\alpha \in(0,1)$.

Proof. We obtain part i) using the equi-continuity of the family of operators $\left\{K_{\varepsilon}\right.$ : $\left.W^{1, p} \rightarrow W^{1, q}\right\}_{\varepsilon \geq 0}$ for $p \in(1,2)$ and $q=\frac{2 p}{2-p}$, the uniform boundedness of the $w^{\varepsilon}$ 's in $F_{1, p, T}$, and the fact that $W^{1, q}\left(\mathbb{R}^{2}\right)$ is continuously embedded into $L^{\infty}\left(\mathbb{R}^{2}\right) \cap \mathcal{C}^{1-\frac{2}{q}}$ since $q>2$ (see e.g. 44).

To prove ii) we use the fact that each distributional derivative of the velocity field $K * w$ is obtained by applying some singular integral operator on $w$ (see e.g. Bertozzi and Majda [2, p. 76). We can therefore adapt the arguments in Lemma 2.2 in [10] to check that the operators $\frac{\partial}{\partial x_{i}} K_{j} *: W^{1, r} \rightarrow W^{1, r}$ are continuous. Moreover, using the fact that the Fourier transform of $\frac{\partial}{\partial x_{i}}\left(K_{\varepsilon}\right)_{j}$ is pointwise bounded by that of $\frac{\partial}{\partial x_{i}} K_{j}$, we can follow the lines of Lemma 5.1 in [10] to prove that the family of operators $\frac{\partial}{\partial x_{i}}\left(K_{\varepsilon}\right)_{j} *: W^{1, r} \rightarrow W^{1, r}, \varepsilon \geq 0$ is equi-continuous.

We conclude ii) using the latter, the uniform estimate for $w^{\varepsilon}$ in $F_{1, r,(T ; p)}$ when $r \in\left(2, \frac{2 p}{2-p}\right)$ and the embedding of $W^{1, r}\left(\mathbb{R}^{2}\right)$ into $L^{\infty}\left(\mathbb{R}^{2}\right) \cap \mathcal{C}^{1-\frac{2}{r}}$ for $r>2$.

4.3. Pathwise convergence of the mollified processes. Throughout this section, it is assumed that $\left(\mathbf{H}_{\mathbf{1}}\right)$ and $\left(\mathbf{H}_{\mathbf{p}}\right)$ hold with $p \in\left[\frac{4}{3}, 2\right)$. Let us state the existence and pathwise uniqueness of the nonlinear process related to the vortex equation. The existence is obtained by approximation of the mollified nonlinear processes, when $\varepsilon \rightarrow 0$.

Definition 4.13. We denote by $\mathcal{P}_{p, T}$ the space of probability measures on $C_{T}=$ $[0, T] \times C\left([0, T], \mathbb{R}^{2}\right)$ such that for each $t \in[0, T]$, the signed measure $\tilde{P}_{t}$ has a density $\tilde{\rho}_{t}$ with respect to the Lebesgue measure and $\tilde{\rho} \in F_{0, p, T} \cap F_{0,1, T}$. 
Theorem 4.14. Consider a $\mathbb{R}^{2}$-Brownian motion $B$ and a random variable $\left(\tau, X_{0}\right)$ in $[0, T] \times \mathbb{R}^{2}$ with law $P_{0}$ and independent of the Brownian motion.

a) There exists in $\mathcal{P}_{p, T}$ a unique solution $P$ to the nonlinear martingale problem (MP). The corresponding function $\tilde{\rho}$ is the unique solution of the mild equation (5) in the space $F_{0, p, T} \cap F_{0,1, T}$.

b) There is a unique pathwise solution $((\tau, X), P)$ of the nonlinear s.d.e. $(\mathbf{E})$ :

i) The law $P$ of $(\tau, X)$ belongs to $\mathcal{P}_{p, T}$ and $\tilde{P}_{t}(d x)=\tilde{\rho}_{t}(x) d x$.

$$
\text { ii) } X_{t}=X_{0}+\sqrt{2 \nu} \int_{0}^{t} \mathbf{1}_{\{s \geq \tau\}} d B_{s}+\int_{0}^{t} \mathbf{1}_{\{s \geq \tau\}} K * \tilde{\rho}_{s}\left(X_{s}\right) d s \text {. }
$$

c) For each $\varepsilon>0$, let $X^{\varepsilon}$ be the mollified nonlinear processes constructed in the same probability space as $B$ and $\left(\tau, X_{0}\right)$. Then, for each $r \in\left(p, \frac{2 p}{2-p}\right)$,

$$
E\left(\sup _{t \leq T}\left|X_{t}^{\varepsilon}-X_{t}\right|\right) \leq C(p, r) \varepsilon^{2\left(\frac{1}{p}-\frac{1}{r}\right)} .
$$

Proof. The existence part of a) easily follows from b) by writing Itô's formula for $f\left(t, X_{t}\right)$ and using Remark 2.8. On the other hand, by Lemma 2.7 for any solution $P \in \mathcal{P}_{p, T}$ the associated $\tilde{\rho}$ is a solution of the weak equation. The fact that it also is a mild solution then follows from Remark 2.4, observing that

$$
\int_{0}^{T} \int_{\mathbb{R}^{2}}\left|K * \tilde{\rho}_{s}(x)\right|\left|\tilde{\rho}_{s}(x)\right| d x d s<\infty
$$

by Hölder's inequality since $\tilde{\rho} \in F_{0, \frac{4}{3}, T}$ (by interpolation) and $K * \tilde{\rho} \in F_{0,4, T}$ by Lemma 4.1. Theorem 4.6 then implies that $\tilde{\rho}$ is uniquely determined, and so $P$ indeed solves a linear martingale problem. By Remark 2.8, it is also a weak solution to a (linear) stochastic differential equation. Its pathwise uniqueness is proved below, and this implies the uniqueness of $P$.

The rest of the proof consists of several steps:

Pathwise uniqueness for $(\mathbf{E})$. Let $\left(\tau, Z^{1}\right)$ and $\left(\tau, Z^{2}\right)$ be two pathwise solutions of $(\mathbf{E})$, with laws respectively denoted by $P^{1}, P^{2} \in \mathcal{P}_{p, T}$. As in the previous step we obtain that $\tilde{\rho}^{1}=\tilde{\rho}^{2}=w$ is the unique solution of (5) in the space $F_{0, p, T} \cap F_{0,1, T}$. Therefore, $\left(\tau, Z^{1}\right)$ and $\left(\tau, Z^{2}\right)$ are both solutions of an s.d.e. $\left(\mathbf{E}^{w}\right)$ defined like $(\mathbf{E})$, but with the known drift coefficient $K * w_{s}$ instead of $K * \tilde{\rho}_{s}^{1}$ or $K * \tilde{\rho}_{s}^{2}$ in (35).

By Corollary 4.12 ii), we get for all $r \in\left(2, \frac{2 p}{2-p}\right)$ and $t \leq T$ that

$$
\begin{aligned}
E\left(\sup _{u \leq t}\left|Z_{u}^{1}-Z_{u}^{2}\right|\right) & =E\left(\sup _{u \leq t}\left|\int_{0}^{u}\left(K * w_{s}\left(Z_{s}^{1}\right)-K * w_{s}\left(Z_{s}^{2}\right)\right) d s\right|\right) \\
& \leq \int_{0}^{t} s^{\frac{1}{r}-\frac{1}{p}-\frac{1}{2}} E\left(\sup _{u \leq s}\left|Z_{u}^{1}-Z_{u}^{2}\right|\right) d s .
\end{aligned}
$$

By Lemma 1.1, we conclude that $E\left(\sup _{t \leq T}\left|Z_{t}^{1}-Z_{t}^{2}\right|\right)=0$.

Pathwise convergence. Let $q$ be defined by $\frac{1}{q}=\frac{1}{p}-\frac{1}{2}$. We choose $r \in\left(p, \frac{2 p}{2-p}\right)$ and $l \in(1,2)$ such that $\frac{1}{p}-\frac{1}{2}=\frac{1}{q}=\frac{1}{r}+\frac{1}{l}-1$. We first prove the following estimate: for each $\varepsilon>\varepsilon^{\prime} \geq 0$,

$$
E\left(\int_{0}^{T}\left|K_{\varepsilon} * \tilde{\rho}_{s}^{\varepsilon}\left(X_{s}^{\varepsilon}\right)-K_{\varepsilon^{\prime}} * \tilde{\rho}_{s}^{\varepsilon^{\prime}}\left(X_{s}^{\varepsilon}\right)\right| d s\right) \leq C \varepsilon^{\frac{2-l}{l}} .
$$


Write $\frac{1}{q^{*}}=1-\frac{1}{q}$ and observe that $q^{*} \in[1, p]$ so that by Lemma 3.9 ,

$$
\sup _{\varepsilon>0}\left\|\rho^{\varepsilon}\right\|_{0, q^{*}, T}<\infty .
$$

Since $\rho_{t}^{\varepsilon}$ is the density of $X_{t}^{\varepsilon}$, we get for the left hand side of (38) that

$$
\begin{aligned}
& \int_{0}^{T} E\left|K_{\varepsilon} * \tilde{\rho}_{s}^{\varepsilon}\left(X_{s}^{\varepsilon}\right)-K_{\varepsilon^{\prime}} * \tilde{\rho}_{s}^{\varepsilon^{\prime}}\left(X_{s}^{\varepsilon}\right)\right| d s \leq \int_{0}^{T}\left\|K_{\varepsilon} * \tilde{\rho}_{s}^{\varepsilon}-K_{\varepsilon^{\prime}} * \tilde{\rho}_{s}^{\varepsilon^{\prime}}\right\|_{q}\left\|\rho_{s}^{\varepsilon}\right\|_{q^{*}} d s \\
& \leq C \int_{0}^{T}\left\|K_{\varepsilon} * \tilde{\rho}_{t}^{\varepsilon}-K_{\varepsilon^{\prime}} * \tilde{\rho}_{t}^{\varepsilon}\right\|_{q} \\
&+\left\|K_{\varepsilon^{\prime}} * \tilde{\rho}_{t}^{\varepsilon}-K_{\varepsilon^{\prime}} * \tilde{\rho}_{t}^{\varepsilon^{\prime}}\right\|_{q} d s .
\end{aligned}
$$

By Young's inequality, Lemma 4.4, and Lemma 4.3 iv) the first term in the last integral is bounded by

$$
\left\|K_{\varepsilon}-K_{\varepsilon^{\prime}}\right\| l\left\|\tilde{\rho}_{t}^{\varepsilon}\right\|_{r} \leq C \varepsilon^{\frac{2-l}{l}}\left\|\tilde{\rho}_{t}^{\varepsilon}\right\|_{r} \leq C \varepsilon^{\frac{2-l}{l}} t^{\frac{1}{r}-\frac{1}{p}} .
$$

By Lemma 4.1 and Proposition 4.5 the second term is bounded by $C \varepsilon^{\frac{2-l}{l}}$. Since $\frac{1}{r}-\frac{1}{p}+1>0$, (38) follows. Next, for $u \leq T$, we have

$$
\begin{aligned}
& E\left(\sup _{v \leq u}\left|X_{v}^{\varepsilon}-X_{v}^{\varepsilon^{\prime}}\right|\right) \leq E\left(\int_{0}^{u}\left|K_{\varepsilon} * \tilde{\rho}_{s}^{\varepsilon}\left(X_{s}^{\varepsilon}\right)-K_{\varepsilon^{\prime}} * \tilde{\rho}_{s}^{\varepsilon^{\prime}}\left(X_{s}^{\varepsilon^{\prime}}\right)\right| d s\right) \\
& \leq \int_{0}^{u}\left(E\left|K_{\varepsilon} * \tilde{\rho}_{s}^{\varepsilon}\left(X_{s}^{\varepsilon}\right)-K_{\varepsilon^{\prime}} * \tilde{\rho}_{s}^{\varepsilon^{\prime}}\left(X_{s}^{\varepsilon}\right)\right|+E\left|K_{\varepsilon^{\prime}} * \tilde{\rho}_{s}^{\varepsilon^{\prime}}\left(X_{s}^{\varepsilon}\right)-K_{\varepsilon^{\prime}} * \tilde{\rho}_{s}^{\varepsilon^{\prime}}\left(X_{s}^{\varepsilon^{\prime}}\right)\right|\right) d s
\end{aligned}
$$

$$
\leq C \varepsilon^{\frac{2-l}{l}}+\int_{0}^{u} s^{\frac{1}{r}-\frac{1}{p}-\frac{1}{2}} E\left(\sup _{v \leq s}\left|X_{v}^{\varepsilon}-X_{v}^{\varepsilon^{\prime}}\right|\right) d s
$$

by (38) and Corollary 4.12. We conclude by Lemma 1.1 (since $\frac{1}{r}-\frac{1}{p}-\frac{1}{2}>-1$ ) that

$$
E\left(\sup _{t \leq T}\left|X_{t}^{\varepsilon}-X_{t}^{\varepsilon^{\prime}}\right|\right) \leq C \varepsilon^{\frac{2-l}{l}}
$$

The sequence $\left(X^{\varepsilon}\right)$ is hence Cauchy in the space $L_{T}^{1}:=\left\{Y: E\left(\sup _{t \in[0, T]}\left|Y_{t}\right|\right)<\right.$ $+\infty\}$ and converges therein at speed $C(p, r) \varepsilon^{2\left(\frac{1}{p}-\frac{1}{r}\right)}$ to some process $X^{w}$.

We remark that, although Proposition 4.5 also allows us to obtain an estimate (38) for $l=1$, the last argument yielding estimate (40) does not hold anymore for $r=\frac{2 p}{2-p}$.

The final step is:

Identification of the limit as a solution of $(\mathbf{E})$. Taking $\varepsilon^{\prime}=0$ in the previous estimate we easily get that $\left(\tau, X^{w}\right)$ solves the s.d.e.

$$
X_{t}^{w}=X_{0}+\sqrt{2 \nu} \int_{0}^{t} \mathbf{1}_{\{s \geq \tau\}} d B_{s}+\int_{0}^{t} \mathbf{1}_{\{s \geq \tau\}} K * w_{s}\left(X_{s}^{w}\right) d s
$$

Denote by $P^{w}$ the law of $X^{w}$. We just need to verify that $X^{w}$ is a solution of the nonlinear s.d.e. $(\mathbf{E})$. This amounts to checking that each of the signed measures $\tilde{P}_{t}^{w}$ has a density which is equal to $w$. We have, for each $f \in \mathcal{D}$ and $t \in[0, T]$, that

$$
\begin{aligned}
\left|\int_{\mathbb{R}^{2}} f(x) \tilde{\rho}_{t}^{\varepsilon}(x) d x-\tilde{P}_{t}^{w}(f)\right| & =\left|E\left(f\left(X_{t}^{\varepsilon}\right) h\left(\tau, X_{0}\right) \mathbf{1}_{\{\tau \leq t\}}\right)-E\left(f\left(X_{t}^{w}\right) h\left(\tau, X_{0}\right) \mathbf{1}_{\{\tau \leq t\}}\right)\right| \\
& \leq C\|\nabla f\|_{\infty} E\left|X_{t}^{\varepsilon}-X_{t}^{w}\right| \rightarrow 0 \text { when } \varepsilon \rightarrow 0 .
\end{aligned}
$$


This concludes the proof, since $\int_{\mathbb{R}^{2}} f(x) \tilde{\rho}_{t}^{\varepsilon}(x) d x \rightarrow \int_{\mathbb{R}^{2}} f(x) w_{t}(x) d x$ by Proposition 4.5 .

4.4. The stochastic vortex method. From the results in the previous sections we readily deduce

Corollary 4.15. Let $T>0$ and assume that $\left(\mathbf{H}_{\mathbf{1}}\right)$ and $\left(\mathbf{H}_{\mathbf{p}}\right)$ hold with $p \in\left[\frac{4}{3}, 2\right)$. Consider a sequence $\varepsilon_{n} \rightarrow 0$ in such a way that

$$
\frac{C_{1} \varepsilon_{n}}{\sqrt{n}} \exp \left(C_{2}\left(\left\|w_{0}\right\|_{1}+\|g\|_{1, T}\right)\left(\varepsilon_{n}^{-2}\right) T\right) \rightarrow 0
$$

when $n \rightarrow \infty$, where $C_{1}$ and $C_{2}$ are the positive constants given in Proposition 3.6.

With the notation (3.4), we define for all $n \in \mathbb{N}$ and $i=1, \ldots, n$ the system of particles

$$
Z^{i n}:=X^{i n, \varepsilon_{n}}
$$

and consider on the same probability space the sequence of i.i.d. processes $\left(\bar{X}^{i}\right)_{i \in \mathbb{N}}$, with $\bar{X}^{i}$ the unique strong solution of

$$
\begin{aligned}
& \text { i) the law } P \text { of }\left(\tau^{i}, \bar{X}^{i}\right) \text { belongs to } \mathcal{P}_{p, T} \text { and } \tilde{P}_{t}(d x)=\tilde{\rho}_{t}(x) d x \\
& \text { ii) } \bar{X}_{t}^{i}=X_{0}^{i}+\sqrt{2 \nu} \int_{0}^{t} \mathbf{1}_{\left\{s \geq \tau^{i}\right\}} d B_{s}^{i}+\int_{0}^{t} \mathbf{1}_{\left\{s \geq \tau^{i}\right\}} K * \tilde{\rho}_{s}\left(\bar{X}_{s}^{i}\right) d s .
\end{aligned}
$$

Then, for all $k \in \mathbb{N}$ and any $r \in\left(p, \frac{2 p}{2-p}\right)$, we have

$E\left(\sup _{t \in[0, T]} \sum_{i=1}^{k}\left|Z_{t}^{i n}-\bar{X}_{t}^{i}\right|\right) \leq k C \varepsilon_{n}^{2\left(\frac{1}{p}-\frac{1}{r}\right)}+k \frac{C_{1} \varepsilon_{n}}{\sqrt{n}} \exp \left(C_{2}\left(\left\|w_{0}\right\|_{1}+\|g\|_{1, T}\right)\left(\varepsilon_{n}^{-2}\right) T\right)$

(the constant $C$ depending on $p, r$ and $T$ ).

The following corollary shows the consistency of our method, to approximate the solution of a Navier-Stokes equation with external force. We exhibit a rate of convergence, which had not been proved even in the classic case.

Corollary 4.16. Consider $\alpha \in] 0, \frac{1}{2}\left[\right.$ and the sequence $\left(\varepsilon_{n}\right)$ given by

$$
\varepsilon_{n}:=\left(\frac{C_{2}\|h\|_{\infty} T}{\alpha \ln n}\right)^{\frac{1}{2}},
$$

with a constant $C_{2}>0$ as in Corollary 4.15, Consider moreover the weighted empirical process

$$
\tilde{\mu}_{s}^{n, \varepsilon_{n}}=\frac{1}{n} \sum_{j=1}^{n} h\left(\tau^{j}, X_{0}^{j}\right) \mathbf{1}_{\left\{s \geq \tau^{j}\right\}} \delta_{Z_{s}^{j n}}
$$

and the approximate velocity field

$$
K_{\varepsilon_{n}} * \tilde{\mu}_{s}^{n, \varepsilon_{n}}(z)=\frac{1}{n} \sum_{j=1}^{n} h\left(\tau^{j}, X_{0}^{j}\right) \mathbf{1}_{\left\{s \geq \tau^{j}\right\}} K_{\varepsilon_{n}}\left(z-Z_{s}^{j n}\right) .
$$

Then, under the assumptions of Theorem 4.14, for all $l \in(1,2)$ we have

$$
\sup _{x \in \mathbb{R}^{2}} E\left(\sup _{t \in[0, T]} t^{\frac{1}{l}}\left|K_{\varepsilon_{n}} * \tilde{\mu}_{t}^{n, \varepsilon_{n}}(x)-u(t, x)\right|\right) \leq C(l, \alpha, T)\left(\frac{\ln n}{n^{\frac{1}{2}-\alpha}}+\frac{1}{(\alpha \ln n)^{\frac{2-l}{2 l}}}\right) .
$$


Proof. First we prove that for all $l \in(1,2)$, for some constant $C(T)$ depending on $l$ and $T$, it holds that for $p \in[4 / 3,2)$,

$$
\sup _{t \leq T} t^{\frac{1}{2}}\left\|w_{t}^{\varepsilon}-w_{t}\right\|_{W^{1, p}} \leq C(T) \varepsilon^{\frac{2-l}{l}} .
$$

From Lemma 4.8 ii), for $\varepsilon \geq 0$ the function $\nabla w^{\varepsilon}$ satisfies

$$
\begin{aligned}
\frac{\partial w_{t}^{\varepsilon}}{\partial x_{i}}(x)= & \frac{\partial}{\partial x_{i}} G_{t}^{\nu} * w_{0}(x)+\frac{\partial}{\partial x_{i}} \int_{0}^{t} G_{t-s}^{\nu} * g_{s}(x) d s \\
& +\int_{0}^{t} \int_{\mathbb{R}^{2}} \frac{\partial G_{t-s}^{\nu}}{\partial x_{i}}(x-y)\left(K_{\varepsilon} * w_{s}^{\varepsilon}\right)(y) \cdot \nabla w_{s}^{\varepsilon}(y) d y d s,
\end{aligned}
$$

since $\operatorname{div} K_{\varepsilon} * w_{s}^{\varepsilon}=0$. Proceeding as in Proposition 4.5, we deduce for $r \in\left(p, \frac{2 p}{2-p}\right)$ given by $\frac{1}{p}-\frac{1}{2}=\frac{1}{r}+\frac{1}{l}-1$ that

$$
\begin{aligned}
\left\|\nabla w_{t}^{\varepsilon}-\nabla w_{t}\right\|_{p} \leq \quad & C \int_{0}^{t}(t-s)^{-\frac{1}{p}}\left(\left\|\left(K_{\varepsilon} * w_{s}^{\varepsilon}\right) \nabla w_{s}^{\varepsilon}-\left(K * w_{s}^{\varepsilon}\right) \nabla w_{s}^{\varepsilon}\right\|_{\frac{2 p}{4-p}}\right. \\
& +\left\|\left(K * w_{s}^{\varepsilon}\right) \nabla w_{s}^{\varepsilon}-\left(K * w_{s}\right) \nabla w_{s}^{\varepsilon}\right\|_{\frac{2 p}{4-p}} \\
& +\|\left(K * w_{s}\right)\left(\nabla w_{s}^{\varepsilon}-\nabla w_{s} \|_{\frac{2 p}{4-p}}\right) d s \\
\leq \quad & C\left\|w^{\varepsilon}\right\|_{0, p, T}\left\|K-K_{\varepsilon}\right\|_{l}\left\|w^{\varepsilon}\right\|_{1, r,(T ; p)} \int_{0}^{t}(t-s)^{-\frac{1}{p}} s^{\frac{1}{r}-\frac{1}{p}-\frac{1}{2}} d s \\
& +C\left\|w^{\varepsilon}\right\|_{1, p, T} \int_{0}^{t}(t-s)^{-\frac{1}{p}} s^{-\frac{1}{2}}\left\|w_{s}^{\varepsilon}-w_{s}\right\|_{p} d s \\
& +C\|w\|_{0, p, T} \int_{0}^{t}(t-s)^{-\frac{1}{p}}\left\|\nabla w_{s}^{\varepsilon}-\nabla w_{s}\right\|_{p} d s .
\end{aligned}
$$

Notice that $\frac{1}{r}-\frac{1}{p}+\frac{1}{2}>0$. With the help of Lemma 4.4 and Proposition 4.5 (since $\tilde{\rho}^{\varepsilon}=w^{\varepsilon}$ ), we deduce for all $\theta \leq T$ and $t \leq \theta$ that

$t^{\frac{1}{2}}\left\|\nabla w_{t}^{\varepsilon}-\nabla w_{t}\right\|_{p} \leq C \varepsilon^{\frac{2-l}{l}} t^{-\frac{2}{p}+\frac{1}{r}+1}+C \varepsilon^{\frac{2-l}{l}} t^{1-\frac{1}{p}}+t^{1-\frac{1}{p}} C\left\{\sup _{s \leq \theta} s^{\frac{1}{2}}\left\|\nabla w_{s}^{\varepsilon}-\nabla w_{s}\right\|_{p}\right\}$,

where all powers of $t$ are nonnegative. It follows that

$$
\left\{\sup _{s \leq \theta} s^{\frac{1}{2}}\left\|\nabla w_{s}^{\varepsilon}-\nabla w_{s}\right\|_{p}\right\} \leq C(T) \varepsilon^{\frac{2-l}{l}}+C \theta^{1-\frac{1}{p}}\left\{\sup _{s \leq \theta} s^{\frac{1}{2}}\left\|\nabla w_{s}^{\varepsilon}-\nabla w_{s}\right\|_{p}\right\},
$$

from where $\sup _{s \leq \theta_{0}} s^{\frac{1}{2}}\left\|\nabla w_{s}^{\varepsilon}-\nabla w_{s}\right\|_{p} \leq C(T) \varepsilon^{\frac{2-l}{l}}$ for $\theta_{0}>0$ small enough.

By similar steps as before, starting from the equations satisfied by $w_{\cdot+\theta_{0}}^{\varepsilon}$ and $w \cdot+\theta_{0}$, and noting that these functions and their gradients are bounded in $F_{0, p, T-\theta_{0}}$ and $F_{0, r,(T ; p)}$ uniformly in $\varepsilon \geq 0$, we now obtain

$$
\begin{aligned}
\left\|\nabla w_{\theta_{0}+t}^{\varepsilon}-\nabla w_{\theta_{0}+t}\right\|_{p} \leq & C \varepsilon^{\frac{2-l}{l}}+C \varepsilon^{\frac{2-l}{l}} \int_{0}^{t}(t-s)^{-\frac{1}{p}} s^{\frac{1}{r}-\frac{1}{p}} d s \\
& +C \int_{0}^{t}(t-s)^{-\frac{1}{p}}\left\|w_{\theta_{0}+s}^{\varepsilon}-w_{\theta_{0}+s}\right\|_{p} d s \\
& +C \int_{0}^{t}(t-s)^{-\frac{1}{p}}\left\|\nabla w_{\theta_{0}+s}^{\varepsilon}-\nabla w_{\theta_{0}+s}\right\|_{p} d s .
\end{aligned}
$$


Proposition 4.5 and Lemma 1.1 imply that $\sup _{t \leq T-\theta_{0}}\left\|\nabla w_{\theta_{0}+t}^{\varepsilon}-\nabla w_{\theta_{0}+t}\right\|_{p} \leq$ $C(T) \varepsilon^{\frac{2-l}{l}}$. From the previous estimates and Proposition 4.5 we get $\left\|w^{\varepsilon}-w\right\|_{1, p, T} \leq$ $C \varepsilon^{\frac{2-l}{l}}$, from where (43) follows. Next, we have

$$
\begin{aligned}
\left|K_{\varepsilon_{n}} * \tilde{\mu}_{t}^{n, \varepsilon_{n}}(x)-u(t, x)\right| \\
\leq\left|K_{\varepsilon_{n}} * \tilde{\mu}_{t}^{n, \varepsilon_{n}}(x)-\frac{1}{n} \sum_{i=1}^{n} K_{\varepsilon_{n}}\left(\bar{X}_{t}^{i, \varepsilon_{n}}-x\right) h\left(\tau^{i}, X_{0}^{i}\right) \mathbf{1}_{\left\{s \geq \tau^{i}\right\}}\right| \\
\quad+\left|\frac{1}{n} \sum_{i=1}^{n} K_{\varepsilon_{n}}\left(\bar{X}_{t}^{i, \varepsilon_{n}}-x\right) h\left(\tau^{i}, X_{0}^{i}\right) \mathbf{1}_{\left\{s \geq \tau^{j}\right\}}-K_{\varepsilon_{n}} * w_{t}^{\varepsilon_{n}}(x)\right| \\
\quad+\left|K_{\varepsilon_{n}} * w_{t}^{\varepsilon_{n}}(x)-K * w_{t}(x)\right| .
\end{aligned}
$$

Let $L_{\varepsilon_{n}}$ and $M_{\varepsilon_{n}}$, respectively, be a Lipschitz constant for $K_{\varepsilon_{n}}$ and an upper bound for its sup-norm, and $C>0$ a constant such that $M_{\varepsilon_{n}} \leq \frac{C}{\varepsilon_{n}^{2}}$ and $L_{\varepsilon_{n}} \leq \frac{C}{\varepsilon_{n}^{3}}$ for $n$ large enough. By the choice of $\left(\varepsilon_{n}\right)$, and Proposition [3.6, the expectation of the first term is bounded by

$$
L_{\varepsilon_{n}} C_{1} \frac{\varepsilon_{n}}{\sqrt{n}} \exp \left(C_{2}\|h\|_{\infty}\left(\varepsilon_{n}^{-2}\right) T\right) \leq C \frac{n^{\alpha}}{\sqrt{n} \varepsilon_{n}^{2}} \leq C \frac{\alpha \ln n}{\|h\|_{\infty} T n^{\frac{1}{2}-\alpha}},
$$

where $\|h\|_{\infty}=\left\|w_{0}\right\|_{1}+\|g\|_{1, T}$. On the other hand, independence of the processes $\left(\tau^{i}, X^{i \varepsilon_{n}}\right)$ implies that the expectation of the second term on the r.h.s. is bounded above by

$$
\frac{1}{\sqrt{n}} 2 M_{\varepsilon_{n}}\|h\|_{\infty} \leq C \frac{\alpha \ln n}{T n^{\frac{1}{2}}}
$$

For the last term in (47), notice that by similar arguments as in the proof Corollary 4.12 ,

$$
\begin{aligned}
&\left|K_{\varepsilon_{n}} * w_{t}^{\varepsilon_{n}}(x)-K * w_{t}(x)\right| \leq C\left\|K_{\varepsilon_{n}} * w_{t}^{\varepsilon_{n}}-K_{\varepsilon_{n}} * w_{t}\right\|_{W^{1, \frac{2 p}{2-p}}} \\
&+C\left\|K_{\varepsilon_{n}} * w_{t}-K * w_{t}\right\|_{W^{1, \frac{2 p}{2-p}}} \\
& \leq C\left\|w_{t}^{\varepsilon_{n}}-w_{t}\right\|_{W^{1, p}}+\left\|K_{\varepsilon_{n}}-K\right\|_{l}\left\|w_{t}\right\|_{W^{1, r}} \\
& \leq C \varepsilon_{n}^{\frac{2-l}{l}} t^{-\frac{1}{2}}+C \varepsilon_{n}^{\frac{2-l}{l}} t^{\frac{1}{r}-\frac{1}{p}-\frac{1}{2}}\|w\|_{1, r,(T ; p)},
\end{aligned}
$$

where $l$ and $r$ are chosen as before. Since $\frac{1}{r}-\frac{1}{p}-\frac{1}{2}=\frac{1}{l}$, we conclude that

$$
\begin{aligned}
\sup _{x \in \mathbb{R}^{2}} E\left(\sup _{t \in[0, T]} t^{\frac{1}{l}}\left|K_{\varepsilon_{n}} * \tilde{\mu}_{t}^{n, \varepsilon_{n}}(x)-u(t, x)\right|\right) & \leq C\left(\frac{\ln n}{n^{\frac{1}{2}-\alpha}}+\varepsilon_{n}^{\frac{2-l}{l}}\right) \\
& \leq C(l, \alpha, T)\left(\frac{\ln n}{n^{\frac{1}{2}-\alpha}}+\frac{1}{(\alpha \ln n)^{\frac{2-l}{2 l}}}\right)
\end{aligned}
$$




\section{Extension to $L^{1}$ InItial CONDITION AND ForCE FIELD}

In this section, we will remove the $L^{p}, p>1$, assumption on the data, and present the extension of the previous results to this setting.

5.1. Analytical results. The analytical part can be done to a large extent by adapting some arguments developed by Ben-Artzi [1] and Brezis [5] (in the absence of external fields). Thus, we shall only present in detail arguments that are different from those works or from previous sections.

A key point for following [1] and [5] is the continuity in time of mild solutions. This is why we will need the following hypothesis, assumed throughout the sequel:

$\left(\mathbf{H}_{\mathbf{1}}^{\prime}\right)$ :

- $w_{0} \in L^{1}\left(\mathbb{R}^{2}\right)$ and

- $g \in C\left([0, T], L^{1}\left(\mathbb{R}^{2}\right)\right)$.

For $p \in[1, \infty]$ and a measurable functions $v: \mathbb{R}^{2} \times[0, T] \rightarrow \mathbb{R}$ define

- $\|v\|_{0, p, T}^{\sharp}=\sup _{0 \leq t \leq T}\left\{t^{1-\frac{1}{p}}\left\|v_{t}\right\|_{p}\right\}$,

- $\|v\|_{1, p, T}^{\sharp}=\sup _{0 \leq t \leq T}\left\{t^{1-\frac{1}{p}}\left\|v_{t}\right\|_{p}+t^{\frac{3}{2}-\frac{1}{p}}\left\|\nabla v_{t}\right\|_{p}\right\}$

and let $F_{0, p, T}^{\sharp}$ and $F_{1, p, T}^{\sharp}$ be the associated Banach spaces.

Remark 5.1. i) By Lemma 2.2 we have $G_{t}^{\nu} * w_{0} \in F_{0,1, T} \cap F_{1, p, T}^{\sharp}$ for all $p$, and $(t, x) \mapsto \int_{0}^{t} G_{t-s}^{\nu} * g(s, x) d s$ belongs to $F_{0,1, T} \cap F_{1, p, T}^{\sharp}$ for $p<2$. Furthermore, from $\left(\mathbf{H}_{1}^{\prime}\right)$ for all $r \in[1, p]$ we get that $W_{0} \in C^{\prime}\left([0, T], L^{r}\right)$.

ii) It is not hard to check that for $\frac{4}{3} \leq p<2$ and $\frac{2 p}{4-p} \leq p^{\prime}<\frac{p}{2-p}$,

$$
\mathcal{B}^{\varepsilon}:\left(C\left([0, T], L^{p}\right)\right)^{2} \rightarrow\left(C\left([0, T], L^{p^{\prime}}\right)\right)
$$

is well defined and continuous.

iii) Moreover, proceeding as in Lemma 4.3, one can check that

$$
\sup _{\varepsilon \geq 0}\left\|\mathcal{B}^{\varepsilon}(v, u)\right\|_{0, p^{\prime}, T}^{\sharp} \leq C\|v\|_{0, p, T}^{\sharp}\|u\|_{0, p, T}^{\sharp}
$$

for all $v, u \in F_{0, p, T}^{\sharp}$ and some constant $C>0$.

As a consequence, we have

Lemma 5.2. Assume $\left(\mathbf{H}_{\mathbf{1}}^{\prime}\right)$ and $\left(\mathbf{H}_{\mathbf{p}}\right)$ with $p \in\left[\frac{4}{3}, 2\right)$. Then, for each $\varepsilon \geq 0$ the unique solution $w^{\varepsilon} \in F_{0,1, T} \cap F_{0, p, T}$ of the mild equation (15) belongs to $C\left([0, T], L^{p} \cap L^{1}\right)$.

Proof. Using the continuity estimate of part iii) in Lemma 4.3 with $p=r=r^{\prime}$, we can proceed as in Proposition 4.10 to prove a local existence statement for (15) in the space $C\left([0, T], L^{p}\right)$. From uniqueness in $F_{0, p, T}$ for the equation satisfied by $w^{\varepsilon}(\theta+\cdot)$ we conclude that $w^{\varepsilon} \in C\left([0, T], L^{p}\right)$. By repeatedly using part ii) in Remark 5.1 (and therein choosing adequate values of " $p$ " and " $p$ ") one can deduce from here that $w^{\varepsilon} \in C\left([0, T], L^{1}\right)$. 
The following lemma is the extension to our setting of a key observation in [1].

Lemma 5.3. Let $\Gamma \subseteq L^{1}\left(\mathbb{R}^{2}\right)$ be a pre-compact set and $\Gamma_{T} \subseteq F_{0,1, T}$ a bounded set. For each $p \in(1,2)$ there is an increasing function $\theta \mapsto \delta\left(\theta, p, \Gamma, \Gamma_{T}\right)$, going to 0 with $\theta$ such that

$$
\sup _{\psi \in \Gamma, \phi \in \Gamma_{T}}\left\|G^{\nu} * \psi+\int_{0}^{\cdot} G_{\cdot-s}^{\nu} * \phi(s) d s\right\|_{0, p, \theta}^{\sharp} \leq \delta\left(\theta, p, \Gamma, \Gamma_{T}\right) .
$$

Lemma 5.4. For $\varepsilon \geq 0$ and each initial condition $\psi \in L^{1} \cap L^{p}$ and external field $\phi \in F_{0,1, T} \cap F_{0, p, T}$ let $w^{\varepsilon, \psi, \phi}$ denote the unique solution in $F_{0,1, T} \cap F_{0, p, T}$ of the associated mollified mild equation (15).

Let $\Gamma \subseteq L^{1}$ and $\Gamma_{T} \subseteq F_{0,1, T}$, respectively, be a pre-compact set and a bounded set, such that moreover $\Gamma \subseteq L^{p}\left(\mathbb{R}^{2}\right)$ and $\Gamma_{T} \subseteq F_{0, p, T}$ for some $p \in\left[\frac{4}{3}, 2\right)$. Then, for some $T_{0}>0$

$$
\sup _{\varepsilon \geq 0} \sup _{\psi \in \Gamma, \phi \in \Gamma_{T}}\left\|w^{\varepsilon, \psi, \phi}\right\|_{0, p, \theta}^{\sharp} \leq 2 \delta\left(\theta, p, \Gamma, \Gamma_{T}\right) \text { for all } \theta \leq T_{0} .
$$

Proof. As in Lemma 4.3 iii), with $r=r^{\prime}=p$ one checks that for $t \leq \theta \leq T$,

$$
\left\|\mathcal{B}^{\varepsilon}\left(w^{\varepsilon, \psi, \phi}, w^{\varepsilon, \psi, \phi}\right)_{t}\right\|_{p} \leq C_{p} t^{\frac{1}{p}-1}\left(\left\|w^{\varepsilon, \psi, \phi}\right\|_{0, p, \theta}^{\sharp}\right)^{2} .
$$

With this and Lemma 5.3 we deduce that the function $f_{\theta}(s):=C_{p} s^{2}-s+$ $\delta\left(\theta, p, \Gamma, \Gamma_{T}\right)$ satisfies $f_{\theta}\left(\left\|w^{\varepsilon, \psi, \phi}\right\|_{0, p, \theta}\right) \geq 0$ for $\theta \leq T$. A careful study of $f_{\theta}(s)$ along the lines of [1], pages 350-351, using the continuity of $\theta^{\prime} \mapsto\left\|w^{\varepsilon, \psi, \phi}\right\|_{0, p, \theta^{\prime}}^{\sharp}$ and its convergence to 0 when $\theta^{\prime} \rightarrow 0$ yields the desired result.

We next state the main analytical results needed in the current setting:

Theorem 5.5. Let $p \in\left[\frac{4}{3}, 2\right)$ be fixed and $w_{0}$ and $g$ be functions satisfying $\left(\mathbf{H}_{\mathbf{1}}^{\prime}\right)$.

a) For each $\varepsilon \geq 0$, there exists a unique solution $w^{\varepsilon}$ to the mild vortex equation (15) in the space

$$
\left.\left.C\left([0, T], L^{1}\right) \cap C(] 0, T\right], L^{p}\right) .
$$

Moreover, we have

$$
\sup _{\varepsilon \geq 0}\left\|w^{\varepsilon}\right\|_{0, p, \theta}^{\sharp} \rightarrow 0 \text { when } \theta \rightarrow 0 .
$$

b) We denote $w=w^{0}$. We have $w_{t}^{\varepsilon} \rightarrow w_{t}$ in $L^{p}$ for all $\left.\left.t \in\right] 0, T\right]$, and

$$
\sup _{t \in[0, T]}\left(t^{\frac{1}{2}}\left\|w_{t}^{\varepsilon}-w_{t}\right\|_{p}\right) \leq C(p, T) \varepsilon^{\frac{2-p}{p}} .
$$

Proof. a) We split the proof in several parts.

Uniqueness: We can follow without difficulty the argument of Brezis 5. In our case, we remark that for $w \in C\left([0, T], L^{1}\right)$, the set $\Gamma:=\left\{w_{r}\right\}_{r \in\left[0, \frac{T}{2}\right]} \subseteq L^{1}$ is pre-compact and the set $\Gamma_{T / 2}:=\left\{g_{r+\cdot}(\cdot):[0, T / 2] \times \mathbb{R}^{2} \rightarrow \mathbb{R}\right\}_{r \in[0, T / 2]}$ is bounded in $F_{0,1, \frac{T}{2}}$. Lemma 5.4 provides an increasing function $\delta(\theta)$ not depending on $r \in[0, T /$ $2]$, going to 0 with $\theta$, such that

$$
t^{1-\frac{1}{p}}\left\|w_{r+t}\right\|_{p} \leq \delta(\theta)
$$

for small enough $\theta$ and all $t \in(0, \theta]$. From here on the argument is exactly as in [5]. 
Existence: Let $w_{0}^{n} \in L^{1} \cap L^{p}$ and $g^{n} \in C\left([0, T], L^{1} \cap L^{p}\right)$ be such that $w_{0}^{n} \rightarrow w_{0}$ in $L^{1}$ and $g^{n} \rightarrow g$ in $C\left([0, T], L^{1}\right)$ when $n \rightarrow \infty$. We write $w^{\varepsilon, n}$ for the associated solutions. Then,

$$
\Gamma=\left\{w_{0}^{n}\right\}_{\{n \in \mathbb{N}\}} \text { and } \Gamma_{T}=\left\{g^{n}\right\}_{\{n \in \mathbb{N}\}}
$$

are sets satisfying the hypothesis of Lemma 5.4. By standard $L^{p}$ estimates for $G_{t}^{\nu} * w_{0}^{n}$ and $G_{t-s}^{\nu} * g_{s}^{n}$, and proceeding as in (48), we have

$$
\begin{aligned}
\left\|w_{t}^{\varepsilon, n}-w_{t}^{\varepsilon, m}\right\|_{p} & \leq C t^{\frac{1}{p}-1}\left\|w_{0}^{n}-w_{0}^{m}\right\|_{1}+C t^{\frac{1}{p}}\left\|g^{m}-g^{n}\right\|_{0,1, T} \\
& +C t^{\frac{1}{p}-1}\left(\left\|w^{\varepsilon, n}-w^{\varepsilon, m}\right\|_{0, p, \theta}^{\sharp}\right)^{2}
\end{aligned}
$$

for all $t \in[0, \theta]$. Thanks to Lemma 5.4, for $\theta$ small enough and all $n, m \in \mathbb{N}$,

$$
\begin{aligned}
\left\|w^{\varepsilon, n}-w^{\varepsilon, m}\right\|_{0, p, \theta}^{\sharp} \leq & C\left\|w_{0}^{n}-w_{0}^{m}\right\|_{1}+C T\left\|g^{m}-g^{n}\right\|_{0,1, T} \\
& +C \delta\left(\theta, p, \Gamma, \Gamma_{T}\right)\left\|w^{\varepsilon, n}-w^{\varepsilon, m}\right\|_{0, p, \theta}^{\sharp},
\end{aligned}
$$

and $w^{\varepsilon, n}$ is thus Cauchy in $F_{0, p, T}^{\sharp}$ if $\theta$ is small enough. Then, from the mild equation satisfied by the function $w_{\theta+.}^{\varepsilon, n}(\cdot)$ we get that $\left\{w_{\theta+.}^{\varepsilon, n}\right\}_{n \in \mathbb{N}}$ is Cauchy in the space $F_{0, p, T-\theta}$. Thus, $\left\{w^{\varepsilon, n}\right\}_{n \in \mathbb{N}}$ converges in $F_{0, p, T}^{\sharp}$ to some limit $w^{\varepsilon}$. Continuity of $\mathcal{B}^{\varepsilon}$ in $F_{0, p, T}^{\sharp}$ (cf. Remark 5.1) implies that $w^{\varepsilon}$ solves equation (15).

Continuity of $t \mapsto w_{t}^{\varepsilon} \in L^{1} \cap L^{p}$ on $\left.] 0, T\right]$ : By Lemma [5.2, $t \rightarrow w_{\theta+t}^{\varepsilon, n} \in L^{p}$ is continuous for all $n$, which clearly implies that $\left.\left.w^{\varepsilon} \in C(] 0, T\right], L^{p}\right)$. On the other hand, is is not hard to establish that $\left.\left.\mathcal{B}^{\varepsilon}:\left(F_{0, r, T}^{\sharp} \cap C(] 0, T\right], L^{r}\right)\right)^{2} \rightarrow F_{0, r^{\prime}, T}^{\sharp} \cap$ $\left.C(] 0, T], L^{r^{\prime}}\right)$ is continuous when $\frac{4}{3} \leq r \leq p, \frac{2 r}{4-r} \leq r^{\prime}<\frac{r}{2-r}$, and that $W_{0} \in$ $\left.C(] 0, T], L^{r}\right)$ for all $r \in[1, p]$. By an iterative argument we can deduce that $w^{\varepsilon} \in$ $\left.C(] 0, T], L^{1}\right)$.

Behavior at $\theta=0$ of $\left\|w^{\varepsilon}\right\|_{0, p, \theta}^{\sharp}$ : From Remark [5.1 iii) we deduce for each $\frac{4}{3} \leq r \leq p, \frac{2 r}{4-r} \leq r^{\prime}<\frac{r}{2-r}$, and $\theta \leq T$ that

$$
\left\|w^{\varepsilon}\right\|_{0, r^{\prime}, \theta}^{\sharp} \leq\left\|W_{0}\right\|_{0, r^{\prime}, \theta}^{\sharp}+C_{r, r^{\prime}}\left(\left\|w^{\varepsilon}\right\|_{0, r, \theta}^{\sharp}\right)^{2},
$$

for a constant $C_{r, r^{\prime}}$ not depending on $\varepsilon \geq 0$. By Lemma 5.3, if furthermore $r^{\prime} \neq 1$, we obtain for small enough $\theta>0$ that

$$
\left\|w^{\varepsilon}\right\|_{0, r^{\prime}, \theta}^{\sharp} \leq \delta\left(\theta, r^{\prime}, \Gamma, \Gamma_{T}\right)+C_{r, r^{\prime}}\left(\left\|w^{\varepsilon}\right\|_{0, r, \theta}^{\sharp}\right)^{2} .
$$

Taking $r=r^{\prime}=p$ and proceeding as in Lemma 5.4, we conclude for small enough $\theta$ that

$$
\sup _{\varepsilon \geq 0}\left\|w^{\varepsilon}\right\|_{0, p, \theta}^{\sharp} \leq 2 \delta\left(\theta, p, \Gamma, \Gamma_{T}\right) .
$$

Continuity of $t \mapsto w^{\varepsilon}(t) \in L^{1}$ in $t=0$ : a) By (49), if $\left\|w^{\varepsilon}\right\|_{0, r, \theta}^{\sharp} \rightarrow 0$ when $\theta \rightarrow 0$, then also $\left\|w^{\varepsilon}\right\|_{0, r^{\prime}, \theta}^{\sharp} \rightarrow 0$. An iterative argument using (49), starting from (50) and suitably choosing consequent values of $r$ and $r^{\prime}$, shows that $\left\|w^{\varepsilon}\right\|_{0, \frac{4}{3}, \theta}^{\sharp} \rightarrow 0$. Taking $p^{\prime}=1$ in Remark 5.1 ii) and the value $\frac{4}{3}$ in place of $p$ yields

$$
\left\|w^{\varepsilon}(t)-w_{0}\right\|_{1} \leq\left\|G_{t}^{\nu} * w_{0}-w_{0}\right\|_{1}+t\|g\|_{0,1, T}+C\left(\left\|w^{\varepsilon}\right\|_{0, \frac{4}{3}, t}^{\sharp}\right)^{2} \rightarrow 0 \text { when } t \rightarrow 0 \text {. }
$$

b) Thanks to Remark 5.1 iii), we have that $\sup _{\varepsilon \geq 0}\left\|w^{\varepsilon}\right\|_{0,2, T}^{\sharp}<\infty$. Consequently, taking $l=p$ in Lemma 4.4 and using Young's inequality we obtain, for $\frac{1}{q}=\frac{1}{p}-\frac{1}{2}$, 
that

$$
\left\|K_{\varepsilon} * w_{s}^{\varepsilon}-K * w_{s}^{\varepsilon}\right\|_{q} \leq\left\|K_{\varepsilon}-K\right\|_{p}\left\|w_{s}^{\varepsilon}\right\|_{2} \leq C s^{-\frac{1}{2}} \varepsilon^{\frac{2-p}{p}} .
$$

By standard estimates, the previous considerations and (50), we deduce that for $t \leq \theta$

$$
\begin{aligned}
\left\|w_{t}^{\varepsilon}-w_{t}\right\|_{p} \leq & C \int_{0}^{t}(t-s)^{-\frac{1}{p}}\left\|w_{s}^{\varepsilon}-w_{s}\right\|_{p}\left(\left\|w_{s}^{\varepsilon}\right\|_{p}+\left\|w_{s}\right\|_{p}\right) d s \\
& +C \int_{0}^{t}(t-s)^{-\frac{1}{p}}\left\|K_{\varepsilon} * w_{s}-K * w_{s}\right\|_{q}\left(\left\|w_{s}^{\varepsilon}\right\|_{p}+\left\|w_{s}\right\|_{p}\right) d s \\
\leq & C \delta\left(\theta, p, \Gamma, \Gamma_{T}\right) t^{-\frac{1}{2}} \sup _{s \in[0, \theta]}\left(s^{\frac{1}{2}}\left\|w_{s}^{\varepsilon}-w_{s}\right\|_{p}\right)+C \delta\left(\theta, p, \Gamma, \Gamma_{T}\right) \varepsilon^{\frac{2-p}{p}} t^{-\frac{1}{2}}
\end{aligned}
$$

Therefore,

$$
\sup _{t \in[0, \theta]}\left(t^{\frac{1}{2}}\left\|w_{t}^{\varepsilon}-w_{t}\right\|_{p}\right) \leq C \delta\left(\theta, p, \Gamma, \Gamma_{T}\right)\left[\sup _{t \in[0, \theta]}\left(t^{\frac{1}{2}}\left\|w_{t}^{\varepsilon}-w_{t}\right\|_{p}\right)+\varepsilon^{\frac{2-p}{p}}\right],
$$

and so for some small enough $\theta>0$ we have

$$
\sup _{t \in[0, \theta]}\left(t^{\frac{1}{2}}\left\|w_{t}^{\varepsilon}-w_{t}\right\|_{p}\right) \leq C \delta(\theta) \varepsilon^{\frac{2-p}{p}} .
$$

From this estimate and the mild equations satisfied by $w_{\theta+}^{\varepsilon}$. and $w_{\theta+}$, and arguing as in Proposition 4.5, we get $\left\|w_{\theta+.}^{\varepsilon}-w_{\theta+\cdot}\right\|_{0, p, T-\theta} \leq C \varepsilon^{\frac{2-p}{p}}$. The last two estimates prove $b)$.

The following result states additional regularity properties of the solutions of the mollified equations, needed to study the martingale problem. We omit the proof, which is similar as in the case of $L^{1} \cap L^{p}$ data, with the help of the same local existence result. Some additional technical difficulties arising can be treated as previously in the current section.

Theorem 5.6. For each $p \in\left[\frac{4}{3}, 2\right)$ we have $\sup _{\varepsilon \geq 0}\left\|w^{\varepsilon}\right\|_{1, p, T}^{\sharp}<\infty$.

By similar arguments as in Corollary $4.12 \mathrm{i}$ ), we deduce that

Corollary 5.7. For the same values of $p$, we have

$$
\sup _{\varepsilon \geq 0} \sup _{t \in[0, T]}\left\{t^{\frac{3}{2}-\frac{1}{p}}\left(\left\|K_{\varepsilon} * w_{t}^{\varepsilon}\right\|_{\infty}+\left\|K_{\varepsilon} * w_{t}^{\varepsilon}\right\|_{\mathcal{C}^{2-\frac{2}{p}}}\right)\right\}<\infty .
$$

5.2. The nonlinear process and particle approximations. Let us now generalize the previous probabilistic interpretation to the $L^{1}$-setting and show weak convergence of the mollified particle system. The explosion of the solution at time 0 will prevent us from obtaining pathwise results.

Definition 5.8. For $p \in\left[\frac{4}{3}, 2\right)$, we denote by $\mathcal{P}_{p, T}^{\prime}$ the space of probability measures on $C_{T}=[0, T] \times C\left([0, T], \mathbb{R}^{2}\right)$ such that for each $t \in[0, T]$, the signed measure $\tilde{P}_{t}$ has a density $\tilde{\rho}_{t}$ with respect to the Lebesgue measure and $\tilde{\rho} \in C\left([0, T], L^{1}\right) \cap$ $\left.C(] 0, T], L^{p}\right) \cap F_{0, p, T}^{\sharp}$. 
Theorem 5.9. Assume $\left(\mathbf{H}_{1}^{\prime}\right)$.

a) For each $p \in\left[\frac{4}{3}, 2\right)$, there exists in the class $\mathcal{P}_{p, T}^{\prime}$ a unique solution $P$ to the nonlinear martingale problem (MP). The corresponding function $\tilde{\rho}$ is equal to the unique solution $\left.\left.w \in C\left([0, T], L^{1}\right) \cap C(] 0, T\right], L^{p}\right) \cap F_{0, p, T}^{\sharp}$ of the mild equation (5).

b) The solution $P \in \mathcal{P}_{p, T}^{\prime}$ is the limit in law when $\varepsilon \rightarrow 0$ of the laws $P_{\varepsilon}$ of the mollified processes $\left(X^{\varepsilon}\right)$.

Proof. We proceed in several steps.

Uniqueness. Let $P \in \mathcal{P}_{p, T}^{\prime}$ be a solution of (MP). By the interpolation inequality $\|f\|_{\frac{4}{3}}^{\frac{4}{3}} \leq\|f\|_{1}+\|f\|_{p}^{p}$ applied to $f=t \tilde{\rho}_{t}$ (and multiplying then by $t^{-1}$ ), we see that $\tilde{\rho} \in F_{0, \frac{4}{3}, T}^{\sharp}$. Therefore, as for (36), we obtain

$$
\int_{[0, T] \times \mathbb{R}^{2}}\left|K * \tilde{\rho}_{t}(x)\right|\left|\tilde{\rho}_{t}(x)\right| d x d t<\infty .
$$

By standard arguments we deduce that $\tilde{\rho}$ is a solution of (5) in the space

$$
\left.\left.C\left([0, T], L^{1}\right) \cap C(] 0, T\right], L^{\frac{4}{3}}\right) \cap F_{0, \frac{4}{3}, T}^{\sharp} .
$$

Consequently, for any two solutions $P^{1}$ and $P^{2}$ of (MP) the associated functions $\tilde{\rho}^{1}$ and $\tilde{\rho}^{2}$ coincide by Theorem [5.5, and we write $w=\tilde{\rho}^{2}=\tilde{\rho}^{1}$. Now define a family $\left(\hat{P}_{t}^{i}\right)_{t \in[0, T]}$ of sub-probability measures $\hat{P}_{t}^{i}$ on $\mathbb{R}^{2}$ by

$$
\int_{\mathbb{R}^{2}} f(x) \hat{P}_{t}^{i}(d x)=E^{P^{i}}\left(f\left(X_{t}\right) \mathbf{1}_{\{\tau \leq t\}}\right)
$$

with $(\tau, X)$ the canonical process. It is not immediate whether each $\hat{P}_{t}^{i}$ has a density. Denote by $D_{n}, n \in \mathbb{N} \backslash\{0\}$, the shift operator in the canonical space $[0, T] \times C\left([0, T], \mathbb{R}^{2}\right)$,

$$
D_{n}((\tau, X))=\left(\left(\tau-\frac{1}{n}\right)_{+}, X_{\cdot+\frac{1}{n}}\right) .
$$

Under $Q_{n}^{i}:=P^{i} \circ D_{n}^{-1}, i=1,2$, the canonical variable $\left(\tau, X_{0}\right)$ has law $P_{\frac{1}{n}}^{i}$ given by

$$
\begin{aligned}
\int_{\mathbb{R}^{2} \times\left[0, T-\frac{1}{n}\right]} f(t, x) P_{\frac{1}{n}}^{i}(d t, d x) & =E^{P^{i}}\left(f\left(\left(\tau-\frac{1}{n}\right)_{+}, X_{\frac{1}{n}}\right)\right) \\
& =\int_{\frac{1}{n}}^{T} \int_{\mathbb{R}^{2}} f\left(t-\frac{1}{n}, x\right) P_{0}(d t, d x)+\int_{\mathbb{R}^{2}} f(0, x) \hat{P}_{\frac{1}{n}}^{i}(d x) .
\end{aligned}
$$

Thus, the canonical process $(\tau, X)$ solves under $Q_{n}^{i}$ the martingale problem

- $Q \circ\left(\tau, X_{0}\right)^{-1}=P_{\frac{1}{n}}^{i}$,

- $f\left(t, X_{t}\right)-f\left(\tau, X_{0}\right)-\int_{0}^{t}\left[\frac{\partial f}{\partial s}\left(s, X_{s}\right)+\nu \triangle f\left(s, X_{s}\right)\right.$

$$
\left.+K * w_{s+\frac{1}{n}}\left(X_{s}\right) \nabla f\left(s, X_{s}\right)\right] \mathbf{1}_{s \geq \tau} d s,
$$

$0 \leq t \leq T-\frac{1}{n}$, is a continuous Q-martingale for all $f \in \mathcal{C}_{b}^{1,2}$

w.r.t. the filtration $\mathcal{G}_{t}=\mathcal{F}_{t+\frac{1}{n}}$.

Notice that if we prove that the "initial condition" $Q_{n}^{i} \circ\left(\tau, X_{0}\right)^{-1}$ is uniquely determined, we deduce that (MP) has a unique solution, remarking first that the 
drift coefficient $K * w_{s+\frac{1}{n}}$ is bounded (Corollary [5.7). Then, we can adapt standard results on martingale problems to deduce that $P^{1} \circ D_{n}^{-1}=P^{2} \circ D_{n}^{-1}$ for all $n \in \mathbb{N}$, and this implies that $P^{1}=P^{2}$.

We therefore proceed to check that $P_{\frac{1}{n}}^{1}=P_{\frac{1}{n}}^{2}$, or equivalently (by (52)), that $\hat{P}_{\frac{1}{n}}^{1}=\hat{P}_{\frac{1}{n}}^{2}$. Observe that for $t>0$ the indicator function in the definition (52) can be replaced by that of the event $\{\tau<t\}$. Thus, it is not hard to check that for $t>\frac{1}{n}$ it holds that

$$
\int f(x) \hat{P}_{t}^{i}(d x)=E^{Q_{n}^{i}}\left(f\left(X_{t-\frac{1}{n}}\right) \mathbf{1}_{\left\{\tau<t-\frac{1}{n}\right\}}\right) .
$$

On the other hand, since $K * w_{s+\frac{1}{n}}$ is bounded, by a standard argument based on Girsanov's theorem we can check that $Q_{n}^{i}$ is absolutely continuous (on $\left[0, T-\frac{1}{n}\right] \times$ $\left.C\left(\left[0, T-\frac{1}{n}\right], \mathbb{R}^{2}\right)\right)$ w.r.t. the law of the process $\left(\tau, X_{0}+\int_{0}^{t} \mathbf{1}_{s \geq \tau} d B_{s}\right)$, where $\left(\tau, X_{0}\right)$ has distribution $P_{\frac{1}{n}}^{i}$ and $B$ is an independent Brownian motion. From this and (54) it follows that $\hat{P}_{t}^{i}(d x)$ has a density (independent of whether $P_{\frac{1}{n}}^{i}$ does or not), and so $\hat{P}_{t}^{i}$ has a density for all $t$.

We denote the density of $\hat{P}_{t}^{i}$ by $\hat{\rho}_{t}^{i}$. We just have to prove that $\hat{\rho}_{t}^{1}=\hat{\rho}_{t}^{2}$.

Following similar arguments as in the proof of Lemma 3.9 ii), and using the fact that

$$
\int_{[0, T] \times \mathbb{R}^{2}}\left|K * w_{t}(x)\right| \hat{\rho}_{t}^{i}(x) d x d t<\int_{0}^{T}\left\|K * w_{t}\right\|_{\infty} d t<\infty
$$

by Corollary [5.7, we deduce that

$$
\hat{\rho}_{t}^{i}(x)=G_{t}^{\nu} * \bar{w}_{0}(x)+\int_{0}^{t} G_{t-s}^{\nu} * \bar{g}_{s}(x) d s+\int_{0}^{t} \nabla G_{t-s}^{\nu} *\left[\left(K * w_{s}\right) \hat{\rho}_{s}^{i}\right](x) d s
$$

for all $t \in[0, T]$, where $\bar{w}_{0}(x)=\frac{\left|w_{0}(x)\right|}{\left\|w_{0}\right\|_{1}+\|g\|_{1, T}}$ and $\bar{g}_{s}(x)=\frac{\left|g_{s}(x)\right|}{\left\|w_{0}\right\|_{1}+\|g\|_{1, T}}$. We take $L^{\frac{4}{3}}$ norm and use the estimate $\left\|K_{\varepsilon} * w(s) \hat{\rho}_{s}^{i}\right\|_{1} \leq C s^{-\frac{3}{4}}$ (following from Corollary 5.7) to get that

$$
\left\|\hat{\rho}_{t}^{i}\right\|_{\frac{4}{3}} \leq C t^{-\frac{1}{4}}+C+C \int_{0}^{t}(t-s)^{-\frac{3}{4}} s^{-\frac{3}{4}} d s=C t^{-\frac{1}{4}}+C+C t^{-\frac{1}{2}} .
$$

From here, $\sup _{t \in[0, T]}\left(t^{\frac{1}{2}}\left\|\hat{\rho}_{t}^{i}\right\|_{\frac{4}{3}}\right)<\infty$, and by similar steps as in Theorem $\left.5.5 \mathrm{~b}\right)$, we obtain

$$
\sup _{t \in[0, \theta]}\left(t^{\frac{1}{2}}\left\|\hat{\rho}_{t}^{1}-\hat{\rho}_{t}^{2}\right\|_{\frac{4}{3}}\right) \leq C \delta(\theta) \sup _{t \in[0, \theta]}\left(t^{\frac{1}{2}}\left\|\hat{\rho}_{t}^{1}-\hat{\rho}_{t}^{2}\right\|_{\frac{4}{3}}\right)
$$

for small enough $\theta$, and $\delta(\theta)$ a function associated to $w$ as in Theorem 5.5 a), satisfying $\delta(\theta) \rightarrow 0$ when $\theta \rightarrow 0$. This yields $\hat{\rho}_{t}^{1}=\hat{\rho}_{t}^{2}$ for small enough $t$, and then for all $t$ by standard arguments.

Estimates for time-marginal laws of $P^{\varepsilon}$. Let $\tilde{\rho}^{\varepsilon}$ be associated with the mollified process $X^{\varepsilon}$, and $\hat{\rho}^{\varepsilon}$ the density of $f \mapsto E\left(f\left(X_{t}^{\varepsilon}\right) \mathbf{1}_{\{\tau \leq t\}}\right)$. For an arbitrary $p \in\left[\frac{4}{3}, 2\right)$, we take the $L^{p}$ norm in the mild equations satisfied by $\tilde{\rho}^{\varepsilon}$. From the fact that $\sup _{t \in[0, T]}\left\|\left(K_{\varepsilon} * \tilde{\rho}_{t}^{\varepsilon}\right) \tilde{\rho}_{t}^{\varepsilon}\right\|_{1}<C(\varepsilon)<\infty$, and using Lemma 2.2 together with Young's inequality, we deduce that

$$
\sup _{t \in[0, T]} t^{1-\frac{1}{p}}\left\|\tilde{\rho}_{t}^{\varepsilon}\right\|_{p}<\infty .
$$


Similarly, starting from the mild equation satisfied by $\hat{\rho}^{\varepsilon}$,

$$
\hat{\rho}_{t}^{\varepsilon}(x)=G_{t}^{\nu} * \bar{w}_{0}(x)+\int_{0}^{t} G_{t-s}^{\nu} * \bar{g}_{s}(x) d s+\int_{0}^{t} \nabla G_{t-s}^{\nu} *\left[\left(K_{\varepsilon} * \tilde{\rho}_{s}^{\varepsilon}\right) \hat{\rho}_{s}^{\varepsilon}\right](x) d s,
$$

and since $\sup _{t \in[0, T]}\left\|\left(K_{\varepsilon} * \tilde{\rho}_{t}^{\varepsilon}\right) \hat{\rho}_{t}^{\varepsilon}\right\|_{1}<C^{\prime}(\varepsilon)<\infty$, we deduce that

$$
\sup _{t \in[0, T]} t^{1-\frac{1}{p}}\left\|\hat{\rho}_{t}^{\varepsilon}\right\|_{p}<\infty .
$$

By standard arguments, the function $\tilde{\rho}^{\varepsilon}(t+\cdot) \in F_{0, p, T-t}$ solves the mollified mild equation with data satisfying $\left(\mathbf{H}_{\mathbf{1}}^{\prime}\right)$ and $\left(\mathbf{H}_{\mathbf{p}}\right)$. From Lemma 5.2 we deduce that $\left.\left.\tilde{\rho}^{\varepsilon} \in C\left([0, T], L^{1}\right) \cap C(] 0, T\right], L^{p}\right)$ and therefore, by Theorem [5.5. $\tilde{\rho}^{\varepsilon}$ equals the unique solution $w^{\varepsilon}$ given therein. In particular, if we define

$$
\tilde{\delta}(\theta):=\sup _{\varepsilon>0} \sup _{t \in[0, \theta]} t^{1-\frac{1}{p}}\left\|\tilde{\rho}_{t}^{\varepsilon}\right\|_{p},
$$

then $\tilde{\delta}(\theta)$ converges to 0 when $\theta$ tends to 0 . Moreover, taking $L^{p}$ norm in (55) and using Remark 5.1 we get

$$
\left\|\hat{\rho}^{\varepsilon}\right\|_{0, p, \theta}^{\sharp} \leq\left\|\bar{W}_{0}\right\|_{0, p, \theta}^{\sharp}+C \tilde{\delta}(\theta)\left\|\hat{\rho}^{\varepsilon}\right\|_{0, p, \theta}^{\sharp}
$$

with $\bar{W}_{0}$ defined in the natural way in terms of $\bar{w}_{0}$ anf $\bar{g}$. It follows that

$$
\left\|\hat{\rho}^{\varepsilon}\right\|_{0, p, \theta_{0}}^{\sharp} \leq 2\left\|\bar{W}_{0}\right\|_{0, p, \theta_{0}}^{\sharp}
$$

for $\theta_{0}>0$ small enough. Since $\sup _{\varepsilon \geq 0}\left\|\tilde{\rho}_{\theta_{0}+\cdot}^{\varepsilon}(\cdot)\right\|_{0, p, T-\theta_{0}}<\infty$ by Theorem 5.5 by looking at the mild equations satisfied by the functions $\hat{\rho}_{\theta_{0}+.}^{\varepsilon}, \varepsilon \geq 0$, which have initial conditions $\hat{\rho}_{\theta_{0}}^{\varepsilon}(x)$ that are bounded in $L^{p}$ uniformly in $\varepsilon$ by (56), we conclude that

$$
\left\|\hat{\rho}^{\varepsilon}\right\|_{0, p, T}^{\sharp}<\infty .
$$

(This estimate will be used below in the particular case $p=\frac{4}{3}$ ).

Tightness of the family $\left(P_{\varepsilon}\right)$. From Corollary [5.7, if $0<\eta<1$ and $S, R$ are stopping times in the filtration of $\left(\tau, X^{\varepsilon}\right)$ such that $S \leq R \leq T$ and $R-S \leq \eta$, we have

$$
\int_{S}^{R}\left|K_{\varepsilon} * \tilde{\rho}_{t}^{\varepsilon}\left(X_{t}^{\varepsilon}\right)\right| d t<C \eta^{\frac{1}{p}-\frac{1}{2}}
$$

for a constant $C>0$ independent of $\varepsilon>0$. Tightness follows from the Aldous criterion $(p<2)$.

Identification of accumulation points as solutions of (MP). Let $P$ be an accumulation point. By suitably approximating the function $h$ by continuous functions (cf. 12]), one can check that

$$
\int_{\mathbb{R}^{2}} \psi(x) \tilde{\rho}_{t}^{\varepsilon}(x) d x=E\left(\psi\left(X_{t}^{\varepsilon}\right) h\left(\tau, X_{0}\right) \mathbf{1}_{\{t \geq \tau\}}\right)
$$

converges to $E^{P}\left(\psi\left(X_{t}\right) h\left(\tau, X_{0}\right) \mathbf{1}_{\{t \geq \tau\}}\right)$ when $\varepsilon$ tends to 0 for every $\psi \in \mathcal{D}$. Consequently, since $\tilde{\rho}^{\varepsilon}=w^{\varepsilon}$, we have by Theorem $5.5 \mathrm{~b}$ ) that

$$
\tilde{P}_{t}(d x)=w_{t}(x) d x
$$

with $w$ the unique solution of the mild vortex equation in $\left.\left.C\left([0, T], L^{1}\right) \cap C(] 0, T\right], L^{p}\right)$. 
Let us take $f \in C_{b}^{1,2}, 0 \leq s_{1} \leq \cdots \leq s_{m} \leq s<t \leq T$, and $\lambda:[0, T] \times \mathbb{R}^{2 m} \rightarrow \mathbb{R}$ a continuous bounded function. To show that $P$ is a solution of (MP), it is enough to prove that

$$
\begin{aligned}
E^{P}\left[\left(\int_{s}^{t}\left\{\frac{\partial f}{\partial r}\left(r, X_{r}\right)+\nu \Delta f\left(r, X_{r}\right)+K * w_{r}\left(X_{r}\right) \nabla f\left(r, X_{r}\right)\right\} \mathbf{1}_{\{r \geq \tau\}} d r\right.\right. & \\
& \left.\left.+f\left(t, X_{t}\right)-f\left(s, X_{s}\right)\right) \times \lambda\left(\tau, X_{s_{1}}, \ldots, X_{s_{m}}\right)\right]=0
\end{aligned}
$$

with $(\tau, X)$ being the canonical process. Define a function $\kappa:[0, T] \times C\left([0, T], \mathbb{R}^{2}\right) \rightarrow$ $\mathbb{R}$ by

$$
\begin{array}{r}
\kappa(\theta, \xi)=\left(\int _ { s } ^ { t } \left\{\frac{\partial f}{\partial r}(r, \xi(r))+\nu \Delta\right.\right. \\
\left.f(r, \xi(r))+K * w_{r}(\xi(r)) \nabla f(r, \xi(r))\right\} \mathbf{1}_{\{r \geq \theta\}} d r \\
+f(t, \xi(t))-f(s, \xi(s))) \times \lambda\left(\theta, \xi\left(s_{1}\right), \ldots, \xi\left(s_{m}\right)\right) .
\end{array}
$$

Thanks to Corollary 5.7, $\kappa$ is continuous and bounded, and consequently,

$$
E^{P}(\kappa(\tau, X))=\lim _{\varepsilon^{\prime} \rightarrow 0} E\left(\kappa\left(\tau, X^{\varepsilon^{\prime}}\right)\right)
$$

for $P^{\varepsilon^{\prime}}$ the subsequence converging to $P$. We conclude by showing that this limit is 0 .

From the martingale problem satisfied by $P^{\varepsilon^{\prime}}$ we deduce that

$$
\begin{aligned}
& \left|E\left(\kappa\left(\tau, X^{\varepsilon^{\prime}}\right)\right)\right| \\
& \quad \leq C E\left[\int_{0}^{T}\left|K_{\varepsilon^{\prime}} * \tilde{\rho}_{s}^{\varepsilon^{\prime}}\left(X_{s}^{\varepsilon^{\prime}}\right)-K * w_{s}\left(X_{s}^{\varepsilon^{\prime}}\right)\right| \mathbf{1}_{\{s \geq \tau\}} d s\right] \\
& \quad \leq C \int_{0}^{T} \int_{\mathbb{R}^{2}}\left|K_{\varepsilon^{\prime}} * \tilde{\rho}_{s}^{\varepsilon^{\prime}}(x)-K * w_{s}(x)\right| \hat{\rho}_{s}^{\varepsilon^{\prime}}(x) d x d s \\
& \quad \leq C \int_{0}^{T}\left(\left\|K_{\varepsilon^{\prime}} * \tilde{\rho}_{s}^{\varepsilon^{\prime}}-K_{\varepsilon^{\prime}} * w_{s}\right\|_{4}+\left\|K_{\varepsilon^{\prime}} * w_{s}-K * w_{s}\right\|_{4}\right)\left\|\hat{\rho}^{\varepsilon^{\prime}}(s)\right\|_{\frac{4}{3}} d s
\end{aligned}
$$

by Hölder's inequality. By Lemma 4.1 with $p=\frac{4}{3}$ and $q=4$, Young's inequality and Lemma 4.4 with $l=\frac{4}{3}$, the latter is bounded above by

$$
\begin{aligned}
C \sup _{\varepsilon>0}\left\|\hat{\rho}^{\varepsilon^{\prime}}\right\|_{0, \frac{4}{3}, T} \int_{0}^{T} s^{-\frac{1}{4}}\left[\| \tilde{\rho}_{s}^{\varepsilon^{\prime}}\right. & \left.-w_{s}\left\|_{\frac{4}{3}}+\right\| K-K_{\varepsilon^{\prime}}\left\|_{\frac{4}{3}}\right\| w_{s} \|_{2}\right] d s \\
& \leq C \int_{0}^{T} s^{-\frac{1}{4}}\left[\left\|\tilde{\rho}_{s}^{\varepsilon^{\prime}}-w_{s}\right\|_{\frac{4}{3}}+\left\|K-K_{\varepsilon^{\prime}}\right\|_{\frac{4}{3}}\left\|w_{s}\right\|_{2}\right] d s,
\end{aligned}
$$

the last inequality owing to (51) and to Theorem $5.5 \mathrm{~b}$ ). Thus, when $\varepsilon^{\prime} \rightarrow 0$, we have

$$
\left|E\left(\kappa\left(\tau, X^{\varepsilon^{\prime}}\right)\right)\right| \leq C\left(\varepsilon^{\prime}\right)^{\frac{1}{2}} \int_{0}^{T} s^{-\frac{1}{4}} s^{-\frac{1}{2}} d s=C T^{\frac{1}{4}}\left(\varepsilon^{\prime}\right)^{\frac{1}{2}} \rightarrow 0 .
$$


Corollary 5.10. Consider a sequence $\varepsilon_{n} \rightarrow 0$ and the system of particles $\left(Z^{i n}\right)_{n \in \mathbb{N}, i=1 \ldots n}$ defined as in Corollary 4.15, Let $p \in\left[\frac{4}{3}, 2\right)$ and $P \in \mathcal{P}^{\prime}{ }_{p, T}$ be the law of the nonlinear processes associated with the unique solution $w \in C\left([0, T], L^{1}\right) \cap$ $\left.C(] 0, T], L^{p}\right)$ of the vortex equation. Then, for each $k \in \mathbb{N}$,

$$
\operatorname{law}\left(Z^{1 n}, \ldots, Z^{k n}\right) \Longrightarrow P^{\otimes k} \quad \text { when } n \rightarrow \infty .
$$

Proposition 5.11. Assume that $\left(\mathbf{H}_{1}^{\prime}\right)$ holds, and recall that $u(t, x)=K * w_{t}(x)$. Let the weighted empirical process $\tilde{\mu}_{s}^{n, \varepsilon_{n}}$ and the sequence $\varepsilon_{n}$ be defined as in Corollary 4.16. Then,

$$
\sup _{x \in \mathbb{R}^{2}} E\left(\left|K_{\varepsilon_{n}} * \tilde{\mu}_{t}^{n, \varepsilon_{n}}(x)-u(t, x)\right|\right) \rightarrow 0
$$

for all fixed $t \in] 0, T]$, as $n$ tends to infinity, from which we deduce that

$$
\int_{0}^{T} \sup _{x \in \mathbb{R}^{2}} E\left(\left|K_{\varepsilon_{n}} * \tilde{\mu}_{t}^{n, \varepsilon_{n}}(x)-u(t, x)\right|\right) d t \rightarrow 0 .
$$

Proof. We follow the proof of Corollary 4.16. Under the weaker assumption $\left(\mathbf{H}_{\mathbf{1}}^{\prime}\right)$, our solution however only belongs to $F_{1, p, T}^{\sharp}$ (see Theorem [5.6), and we are not able to extend the continuity properties of $\mathcal{B}$ used in Corollary 4.16 to some space analogous to $F_{1, r,(T ; p)}$.

We thus consider the family of shifted solutions $\left\{w_{r+}^{\varepsilon}\right\}_{\varepsilon \geq 0}$ for $\left.\left.r \in\right] 0, T\right]$. By similar computations as in (45) we obtain that

$$
\left\|w_{r+\cdot}^{\varepsilon}-w_{r+\cdot}\right\|_{1, p, T} \leq C(r, T) \varepsilon^{\frac{2-l}{l}}+C^{\prime}(r, T) r^{-\frac{1}{2}} \varepsilon^{\frac{2-p}{p}},
$$

where the second term on the r.h.s. is due to the difference of the initial conditions in the shifted versions of (44), controlled by Theorem 5.5 b). From this we deduce that the third term on the r.h.s. of (47) goes to 0 for each fixed $t \in] 0, T]$. As in the proof of Corollary 4.16, the expectations of the two other terms are bounded and go to 0 uniformly in $x \in \mathbb{R}^{2}$ and $t \in[0, T]$. This yields the first convergence result. To apply dominated convergence, we use the fact that $\left\|K_{\varepsilon} * w_{t}^{\varepsilon}\right\|_{\infty} \leq C\left\|w_{t}^{\varepsilon}\right\|_{W^{1, p}}$ and Theorem 5.6 .

\section{Numerical RESUlts}

We assume that $w_{0}$ and $g$ satisfy the moment conditions $\int_{\mathbb{R}^{2}}|x|^{2}\left|w_{0}(x)\right| d x<+\infty$ and $\int_{0}^{T} \int_{\mathbb{R}^{2}}|x|^{2}|g(s, x)| d x d s<+\infty$. Taking $\phi(s, x)=1$ and $\phi(s, x)=|x|^{2}$ in equation (4), and using the fact that $\operatorname{div} K * w(s, x)=0$, we can check that

$$
\int_{\mathbb{R}^{2}} w_{t}(x) d x=\int_{\mathbb{R}^{2}} w_{0}(x) d x+\int_{0}^{t} \int_{\mathbb{R}^{2}} g_{s}(x) d x d s
$$

and

$$
\int_{\mathbb{R}^{2}}|x|^{2} w_{t}(x) d x=\int_{\mathbb{R}^{2}}|x|^{2} w_{0}(x) d x+\int_{0}^{t} \int_{\mathbb{R}^{2}}|x|^{2} g_{s}(x) d x d s+4 \nu \int_{0}^{t} \int_{\mathbb{R}^{2}} w_{s}(x) d x d s .
$$


These quantities are, respectively, called "total flux of vorticity" (TFV) and "moment of fluid impulse" (MFI). Also from equation (4), the "barycenter" $\int_{\mathbb{R}^{2}} x_{i} w_{t}(x) d x$ is preserved.

For the simulations, we take $w_{0}$ to be the centered Gaussian density in $\mathbb{R}^{2}$ with variance $m_{0}=2$, and $g(s, x):=\gamma w_{0}(x)$ with $\gamma \neq 0$ to be fixed. We then have $\left\|w_{0}\right\|_{1}+\|g\|_{1, T}=1+|\gamma| T$.

Consider an equi-spaced partition $\left\{t_{k}\right\}_{k=0}^{N}$ of $[0, T]$. We obtain from (6) that

$$
\left.\left.P(\tau=0)=\frac{1}{1+|\gamma| T} \quad ; \quad P(\tau \in] t_{k-1}, t_{k}\right]\right)=\frac{|\gamma| T}{N(1+|\gamma| T)}
$$

and

$$
\left.\left.P\left(X_{0} \in d x \mid \tau=0\right)=P\left(X_{0} \in d x \mid \tau \in\right] t_{k-1}, t_{k}\right]\right)=w_{0}(x) d x .
$$

Moreover, we have $h(0, x)=1+|\gamma| T$ and $h(t, x)=\operatorname{sign}(\gamma)(1+|\gamma| T)$ for $t \in] 0, T]$.

We take as a parameter $p=P(\tau=0)$. Then, we have $\left.\left.|\gamma|=\frac{1-p}{p T}, P(\tau \in] t_{k-1}, t_{k}\right]\right)$ $=\frac{(1-p)}{N}, h(0, x)=\frac{1}{p}$ and $h(t, x)=\operatorname{sign}(\gamma) \frac{1}{p}$ for $\left.\left.t \in\right] 0, T\right]$. We obtain the expression

$$
\int_{\mathbb{R}^{2}} w_{t}(x) d x=1+\operatorname{sign}(\gamma)\left(\frac{1-p}{p T}\right) t
$$

and

$m(t):=\int_{\mathbb{R}^{2}}|x|^{2} w_{t}(x) d x=2+\left(2 \operatorname{sign}(\gamma)\left(\frac{1-p}{p T}\right)+4 \nu\right) t+2 \nu \operatorname{sign}(\gamma)\left(\frac{1-p}{p T}\right) t^{2}$.

We empirically compute these two quantities at each time $t_{k}$ using the particle vortex method. Choosing $\operatorname{sign}(\gamma)=-1$, we simulate the Euler scheme of the trajectory of each particle $X_{t}^{i, n}=\left(X_{t, 1}^{i, n}, X_{t, 2}^{i, n}\right)$ defined in (16), and obtain

$$
\left(X_{t_{k}, 1}^{i, n}, X_{t_{k}, 2}^{i, n}\right)_{\{1 \leq i \leq n ; 1 \leq k \leq N\}} .
$$

The empirical values of TFV and MFI are given by

$$
\frac{1}{p n} \sum_{i=1}^{n} \mathbf{1}_{\left\{t_{k} \geq \tau^{i}\right\}}\left(\mathbf{1}_{\left\{\tau^{i}=0\right\}}-\mathbf{1}_{\left\{\tau^{i} \neq 0\right\}}\right)
$$

and

$$
M\left(t_{k}\right):=\frac{1}{p n} \sum_{i=1}^{n}\left(\left|X_{t_{k}, 1}^{i, n}\right|^{2}+\left|X_{t_{k}, 2}^{i, n}\right|^{2}\right) \mathbf{1}_{\left\{t_{k} \geq \tau^{i}\right\}}\left(\mathbf{1}_{\left\{\tau^{i}=0\right\}}-\mathbf{1}_{\left\{\tau^{i} \neq 0\right\}}\right)
$$

Since TFV depends only on the number of vortices "alive" at each time and their "sign" (and not on their positions), the first graphic in Figure 1 illustrates the law of large numbers for the random birth time $\tau$. The second graphic in Figure 1 illustrates the preservation of the (null) barycenter. 
TFV and empirical TFV

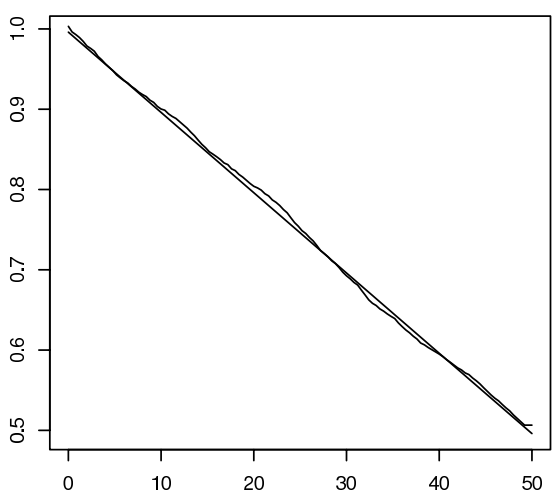

Empirical barycenter (1st coord)

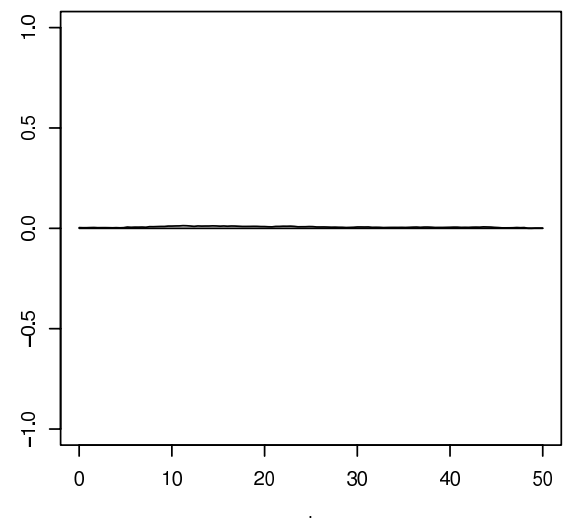

Figure 1. $\varepsilon=10^{-4}, \nu=5 \times 10^{-7}, n=6000, T=50, \Delta t=$ $0.8, p=\frac{2}{3}, \operatorname{sign}(\gamma)=-1$.

Figure 2 shows the theoretical and empirical MFI and the relative error, computed as $\frac{\left|m\left(t_{k}\right)-M\left(t_{k}\right)\right|}{M\left(t_{k}\right)}$. The probabilistic vortex approach seems robust for very small viscosities.
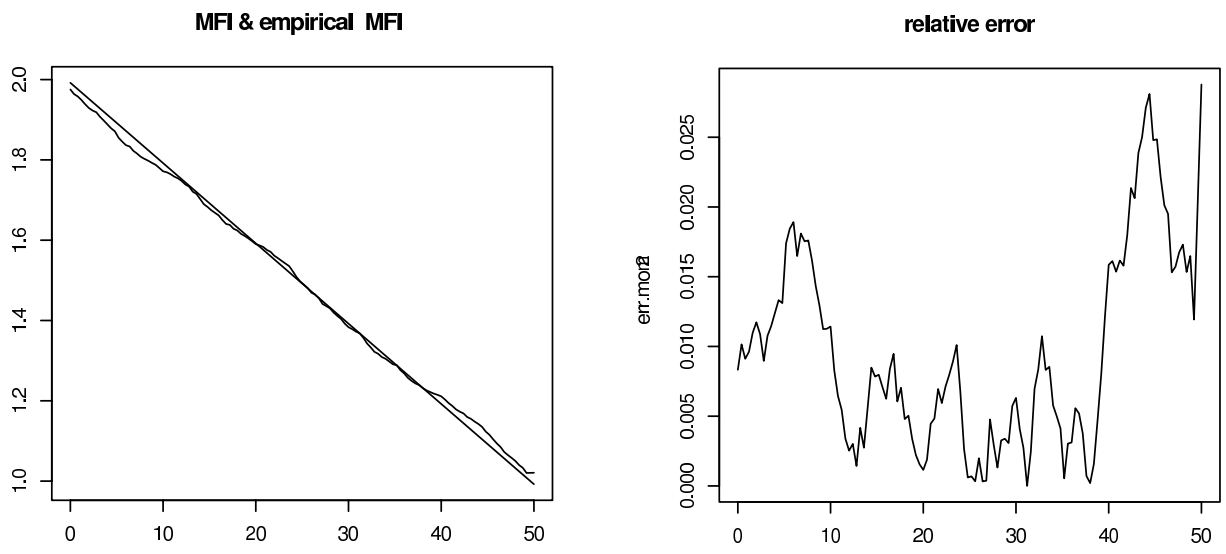

Figure 2. ${ }^{\mathrm{t}} \varepsilon=10^{-4}, \nu=5 \times 10^{-7}, n=6000, T={ }^{\mathrm{t}} 50, \Delta t=$ $0.8, p=\frac{2}{3}, \operatorname{sign}(\gamma)=-1$.

In Figure 3 we show the evolution of the velocity field in a regular grid. At time $t=0$ all vortices have positive sign, and then new vortices with negative signs randomly appear. At each point the norm of the velocity field progressively decreases, attains 0 and then increases, while its direction is progressively reversed. 

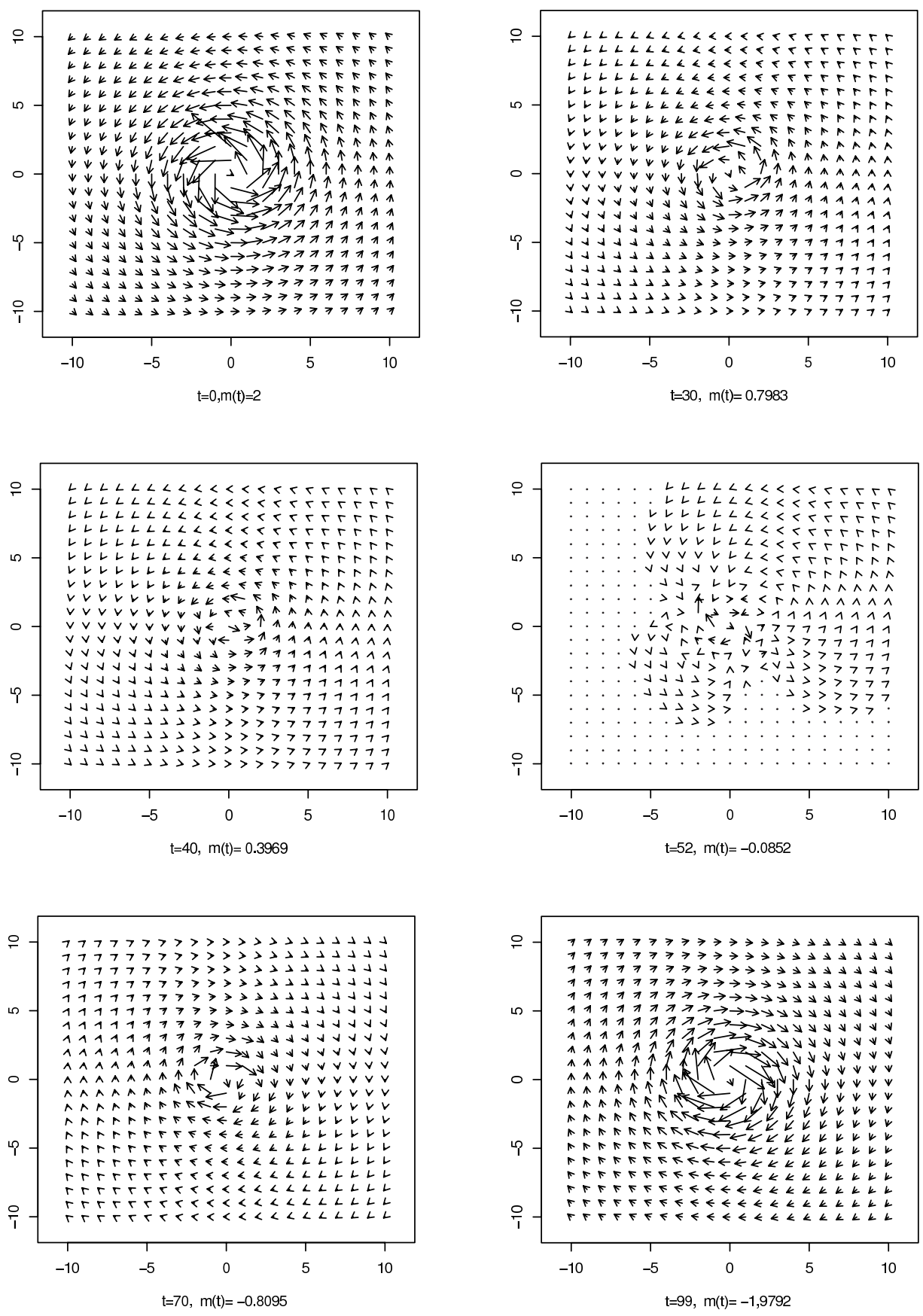

FiguRE 3. $\varepsilon=10^{-4}, \nu=5 \times 10^{-7}, n=1000, T=100, \Delta t=$ $1, p=\frac{1}{3}, \operatorname{sign}(\gamma)=-1$. 


\section{ACKNOWLEDGEMENTS}

We thank Chi Viet Tran and Jaime San Martín for some helpful suggestions about the numerical simulations.

\section{REFERENCES}

1. M. Ben-Artzi, Global solutions of two-dimensional Navier-Stokes and Euler equations, Arch. Anal. Rational Mech. 128 (1994), no. 4, 329-358. MR1308857 (96h:35148)

2. A. Bertozzi and A. Majda, Vorticity and incompressible flow, Cambridge University Press, Cambridge, 2002. MR.1867882 (2003a:76002)

3. M. Bossy, Vitesse de convergence d'algorithmes particulaires stochastiques et application à l'équation de Burgers, Ph.D. thesis, Université de Provence, 1995.

4. H. Brezis, Analyse fonctionelle, Masson, Paris, 1983. MR697382 (85a:46001)

5. — Remarks on the preceding paper by M. Ben-Artzi: Global solutions of twodimensional Navier-Stokes and Euler equations [Arch. Rational Mech. Anal. 128 (1994), no. 4, 329-358], Arch. Rational Mech. Anal. 128 (1994), no. 4, 359-360. MR1308858(96h:35149)

6. B. Busnello, A probabilistic approach to the two dimensional Navier-Stokes equation, Ann. Probab. 27 (1999), 1750-1780. MR1742887 (2001g:60140)

7. M. Cannone, Ondelettes, paraproduits et Navier-Stokes, Diderot Editeur, Paris, 1995. MR 1688096 (2000e:35173)

8. A.J. Chorin and J.E. Marsden, A mathematical introduction to fluid mechanics, Texts in Applied Mathematics 4, Springer, 1993. MR1218879 (94c:76002)

9. G.H. Cottet and P.D. Koumoutsakos, Vortex methods. Theory and practice, Cambridge University Press, Cambridge, 2000. MR1755095 (2001c:76093)

10. J. Fontbona, Nonlinear martingale problems involving singular integrals, J. Funct. Anal. 200 (2003), no. 1, 198-236. MR1974095 (2004b:60225)

11. A. Friedman, Partial differential equations of parabolic type, Prentice-Hall, Inc., 1964. MR 0181836 (31:6062)

12. B. Jourdain, Diffusion processes associated with nonlinear evolution equations for signed measures, Methodol. Comput. Appl. Probab. 2 (2000), no. 1, 69-91. MR.1783154 (2001f:60112)

13. B. Jourdain and S. Méléard, Propagation of chaos and fluctuations for a moderate model with smooth initial data, Ann. Inst. Henri Poincaré Probab. 34 (1998), no. 6, 727-766. MR.1653393 (2000b:60152)

14. - Probabilistic interpretation and particle method for vortex equations with Neumann's boundary condition, Proc. Edinb. Math. Soc. 47 (2004), no. 3, 597-624. MR2097263 (2005i:76033)

15. H. Kunita, Stochastic differential equations and stochastic flows of diffeomorphisms, Ecole d'été de probabilités de Saint-Flour XII-1982, L.N. in Math. 1097, Springer, 1984. MR876080 (87m:60127)

16. C. Marchioro and M.Pulvirenti, Hydrodynamics in two dimensions and vortex theory, Comm. Math. Phys. 84 (1982), no. 4, 483-503. MR667756 (84e:35126)

17. S. Méléard, A trajectorial proof of the vortex method for the 2d Navier-Stokes equation, Ann. Appl. Prob. 10 (2000), no. 4, 1197-1211. MR.1810871 (2002b:76035)

18. Monte-Carlo approximations of the solution of the 2d Navier-Stokes equation with finite measure initial data, Probab. Theory Related Fields. 121 (2001), no. 3, 367-388. MR 1867427 (2003d:76043)

19. P.A. Raviart, An analysis of particle methods, L.N. in Math. 1127, Springer, 1985. MR802214 (87h:76010)

20. E. Stein, Singular integrals and differentiability properties of functions, Princeton University Press, 1970. MR0290095 (44:7280)

21. A.S. Sznitman, Topics in propagation of chaos. Ecole d'été de probabilités de Saint-Flour XIX-1989, L.N. in Math. 1464, Springer, 1991. MR.1108185 (93b:60179)

CMM-Dim, UMi(2807) UChile-CNRS, Universidad de Chile. Casilla 170-3, Correo 3, Santiago-Chile

E-mail address: fontbona@dim.uchile.cl

CMap, ecole polytechnique, CNRS, Route de Saclay 91128 Palaiseau Cedex FRANCE

E-mail address: meleard@cmap.polytechnique.fr 